\title{
Ab initio insight into mechanisms of ozone interaction with a surface of dehydrated nanocrystalline $\mathrm{TiO}_{2}$
}

Ruslan Kevorkyants $^{\mathrm{a}^{*}}$, Yuri V. Chizhov ${ }^{\mathrm{b}}$, and Kirill M. Bulaninª,b

a The Laboratory 'Photoactive Nanocomposite Materials', St. Petersburg State University, ul. Ulyanovskaya 1, St. Petersburg, 198504 Russia

b Photonics Department, St. Petersburg State University, ul. Ulyanovskaya 1, St. Petersburg, 198504 Russia

* Corresponding author’s e-mail: ruslan.kevorkyants@gmail.com; phone: (+7) 9626971903

\section{Supporting information}

Below, please find Cartesian coordinates (angstroms) of the B3PW91//LanL2TZ/6-311g* optimized structures of the monodentate and bidentate ozone adsorption complexes. The data comprise two parts. The first one collects geometries of the singlet complexes, while the second one - of the triplet complexes. Within each part, the complexes are ordered according to ozone adsorption site's number running from 1 to 6 . For each adsorption site, first, the bidentate isomers and then the monodentate isomers are listed. The optimized structures are followed by transition states arising in the reaction $\left[\mathrm{Ti}_{8} \mathrm{O}_{16}-\mathrm{O}_{3}\right]^{\mathrm{T}} \rightarrow \mathrm{Ti}_{8} \mathrm{O}_{17} \mathrm{~S}+\mathrm{O}_{2}{ }^{\mathrm{T}}$. See main text for details.

\section{Optimized structures of the ozone adsorption complexes}

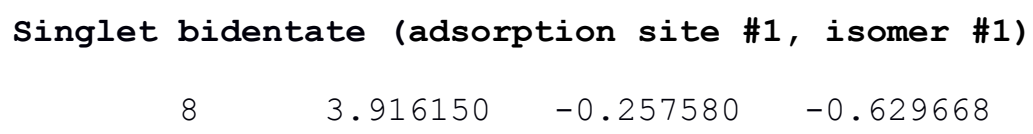




$\begin{array}{rrrr}22 & 2.524730 & 0.829389 & -0.538362 \\ 8 & 1.074266 & -0.196233 & -1.088933 \\ 22 & 0.589912 & -1.913623 & -0.881128 \\ 8 & -1.117117 & -1.961846 & -1.715369 \\ 22 & -2.025943 & -0.931447 & -0.552219 \\ 8 & -2.944611 & -0.813243 & 1.150740 \\ 22 & -2.196425 & 0.618922 & 1.835566 \\ 8 & -1.088201 & 0.640708 & 0.225099 \\ 22 & -2.169534 & 1.917881 & -0.633736 \\ 8 & -2.881300 & 2.129199 & 1.046947 \\ 22 & 3.347841 & -1.813689 & 0.323251 \\ 8 & 4.484767 & -2.626814 & 1.137743 \\ 8 & 2.153942 & -0.706625 & 1.383357 \\ 22 & 0.544886 & 0.026963 & 1.287370 \\ 8 & -0.628213 & 0.379401 & 2.686855 \\ 8 & 2.042108 & -2.859943 & -0.666942 \\ 8 & 1.285096 & 1.682015 & 0.757552 \\ 22 & 0.890354 & 3.084321 & -0.539358 \\ 8 & -0.924991 & 2.964409 & -1.206318 \\ 8 & -0.290481 & -1.613183 & 0.678516 \\ 8 & 1.228046 & 4.537183 & 0.077443 \\ 8 & 2.369587 & 2.304631 & -1.481977 \\ 8 & -2.803903 & 0.419877 & -1.496239 \\ 8 & -2.543959 & -3.357481 & 0.074334 \\ 8 & -3.585566 & -3.377280 & -0.599535 \\ 8 & -3.886627 & -2.288661 & -1.162412\end{array}$

Singlet bidentate (adsorption site \#1, isomer \#2)

$\begin{array}{rrrr}8 & 4.006317 & 0.046283 & -0.706535 \\ 22 & 3.634457 & -1.562040 & 0.259005 \\ 8 & 2.428305 & -2.745257 & -0.701413 \\ 22 & 0.873834 & -1.972419 & -0.882795 \\ 8 & 0.007464 & -1.755042 & 0.692076 \\ 22 & 0.675834 & -0.040544 & 1.285870 \\ 8 & 1.229538 & 1.686569 & 0.748365 \\ 22 & 0.674635 & 3.071002 & -0.506243 \\ 8 & 2.175505 & 2.430854 & -1.514599 \\ 22 & 2.505434 & 0.971556 & -0.589362 \\ 8 & 2.354882 & -0.593395 & 1.352235 \\ 8 & 1.164487 & -0.210607 & -1.102184 \\ 8 & -0.850631 & -2.203632 & -1.668806 \\ 22 & -1.823605 & -1.233257 & -0.549156 \\ 8 & -3.633387 & -2.567901 & -1.029589 \\ 8 & -4.780056 & -2.101812 & -0.708365 \\ 8 & -4.844949 & -0.978279 & -0.222145 \\ 8 & -2.662770 & -1.292977 & 1.198632 \\ 22 & -2.100625 & 0.226066 & 1.886811 \\ 8 & -0.504735 & 0.181522 & 2.710775 \\ 8 & -1.037607 & 0.409126 & 0.262544 \\ 22 & -2.256345 & 1.587142 & -0.550262 \\ 8 & -2.706079 & 0.074851 & -1.490962 \\ 8 & -2.987094 & 1.649874 & 1.138297 \\ 8 & -1.150740 & 2.791804 & -1.094193 \\ 8 & 4.875073 & -2.242588 & 1.042700 \\ 8 & 0.911525 & 4.539969 & 0.120033\end{array}$

Singlet bidentate (adsorption site \#1, isomer \#3) 


$\begin{array}{rrrr}8 & 3.863962 & -0.288497 & -0.910145 \\ 22 & 3.466416 & -1.720362 & 0.292011 \\ 8 & 2.101489 & -2.919843 & -0.398956 \\ 22 & 0.596457 & -2.048514 & -0.563989 \\ 8 & -0.125104 & -1.557092 & 1.024060 \\ 22 & 0.715986 & 0.159384 & 1.323157 \\ 8 & 1.343883 & 1.749545 & 0.513326 \\ 22 & 0.773234 & 2.990147 & -0.878200 \\ 8 & 2.140350 & 2.109794 & -1.896049 \\ 22 & 2.447487 & 0.764174 & -0.805197 \\ 8 & 2.349720 & -0.520978 & 1.333713 \\ 8 & 0.988980 & -0.361087 & -1.050806 \\ 8 & -1.185494 & -2.245406 & -1.190290 \\ 22 & -2.009410 & -1.054261 & -0.127400 \\ 8 & -3.741651 & -2.591825 & -0.217404 \\ 8 & -4.039313 & -2.998923 & -1.392884 \\ 8 & -3.558305 & -2.402867 & -2.346530 \\ 8 & -2.717881 & -0.797253 & 1.635071 \\ 22 & -1.972120 & 0.728991 & 2.093526 \\ 8 & -0.316542 & 0.654582 & 2.790553 \\ 8 & -1.039660 & 0.610563 & 0.378178 \\ 22 & -2.244843 & 1.766021 & -0.484132 \\ 8 & -2.911756 & 0.178401 & -1.133431 \\ 8 & -2.796388 & 2.124142 & 1.233304 \\ 8 & -1.108583 & 2.779102 & -1.293331 \\ 8 & 4.718961 & -2.389987 & 1.066661 \\ 8 & 1.157013 & 4.507282 & -0.481924\end{array}$

\section{Singlet monodentate (adsorption site \#1, isomer \#1)}

$\begin{array}{rrrr}8 & 3.874753 & -0.527527 & -0.916391 \\ 22 & 2.521999 & 0.609478 & -0.826413 \\ 8 & 0.987706 & -0.402281 & -1.094045 \\ 22 & 0.520471 & -2.059968 & -0.587874 \\ 8 & -1.271842 & -2.183342 & -1.171922 \\ 22 & -1.960659 & -0.944269 & -0.057839 \\ 8 & -2.707429 & -0.574564 & 1.682074 \\ 22 & -1.888942 & 0.919036 & 2.094290 \\ 8 & -0.952595 & 0.725313 & 0.390310 \\ 22 & -2.114510 & 1.882868 & -0.521574 \\ 8 & -2.657505 & 2.315138 & 1.188232 \\ 22 & 3.390836 & -1.935120 & 0.280798 \\ 8 & 4.590521 & -2.672571 & 1.077490 \\ 8 & 2.308029 & -0.687998 & 1.299284 \\ 22 & 0.749856 & 0.157236 & 1.320254 \\ 8 & -0.228263 & 0.721770 & 2.788274 \\ 8 & 1.951786 & -3.044631 & -0.412535 \\ 8 & 1.497468 & 1.672741 & 0.492882 \\ 22 & 0.968019 & 2.919849 & -0.919657 \\ 8 & -0.915277 & 2.811125 & -1.350319 \\ 8 & -0.245157 & -1.484497 & 0.979161 \\ 8 & 1.422171 & 4.421536 & -0.541883 \\ 8 & 2.301162 & 1.967293 & -1.921215 \\ 8 & -2.818718 & 0.306209 & -1.120183 \\ 8 & -3.919544 & -2.198843 & -0.240354 \\ 8 & -4.111712 & -2.405769 & -1.483956 \\ 8 & -5.119993 & -3.019157 & -1.795360\end{array}$

Singlet monodentate (adsorption site \#1, isomer \#2) 


$\begin{array}{rrrr}8 & 4.010083 & -0.280053 & -0.718832 \\ 22 & 3.508420 & -1.838870 & 0.264757 \\ 8 & 2.190625 & -2.924162 & -0.665938 \\ 22 & 0.725158 & -1.992581 & -0.847753 \\ 8 & -0.197878 & -1.634987 & 0.700090 \\ 22 & 0.686246 & -0.000341 & 1.298673 \\ 8 & 1.414781 & 1.633174 & 0.712966 \\ 22 & 0.922787 & 3.003996 & -0.597285 \\ 8 & 2.382801 & 2.235621 & -1.581419 \\ 22 & 2.594809 & 0.776875 & -0.624286 \\ 8 & 2.280747 & -0.771635 & 1.323250 \\ 8 & 1.141883 & -0.268196 & -1.122205 \\ 8 & -1.003548 & -2.108708 & -1.600474 \\ 22 & -1.847203 & -1.053970 & -0.421120 \\ 8 & -3.696379 & -2.405543 & -0.850550 \\ 8 & -4.734771 & -1.718053 & -0.584876 \\ 8 & -5.817865 & -2.254931 & -0.758911 \\ 8 & -2.758688 & -0.907219 & 1.292845 \\ 22 & -2.038159 & 0.555310 & 1.931391 \\ 8 & -0.429802 & 0.348595 & 2.734891 \\ 8 & -0.968985 & 0.596865 & 0.297450 \\ 22 & -2.122107 & 1.801447 & -0.565669 \\ 8 & -2.716411 & 0.274839 & -1.373181 \\ 8 & -2.807127 & 2.016931 & 1.133369 \\ 8 & -0.910285 & 2.859606 & -1.198136 \\ 8 & 4.679211 & -2.607919 & 1.074211 \\ 8 & 1.259245 & 4.473137 & -0.020996\end{array}$

Singlet bidentate (adsorption site \#2, isomer \#1)

$\begin{array}{rrrr}8 & -3.934459 & 0.487167 & -0.297912 \\ 22 & -2.345465 & 1.257787 & -0.227157 \\ 8 & -1.396679 & 0.316123 & 1.063334 \\ 22 & -1.336043 & -1.406838 & 1.596286 \\ 8 & 0.023130 & -1.303678 & 2.934914 \\ 22 & 1.344869 & -0.728375 & 1.888277 \\ 8 & 2.693305 & -1.549653 & 0.896659 \\ 22 & 2.641341 & -0.602107 & -0.626275 \\ 8 & 1.140694 & 0.373551 & 0.294214 \\ 22 & 2.276620 & 1.738774 & 0.899449 \\ 8 & 3.458903 & 0.980285 & -0.320243 \\ 22 & -3.558429 & -1.381431 & -0.441456 \\ 8 & -4.613467 & -2.273091 & -1.283757 \\ 8 & -1.878525 & -0.993529 & -1.328209 \\ 22 & -0.238426 & -0.461439 & -0.947357 \\ 8 & 1.326834 & -0.876297 & -1.866005 \\ 8 & -2.871840 & -2.141637 & 1.212643 \\ 8 & -0.707257 & 1.343643 & -1.308717 \\ 22 & -0.301084 & 3.115072 & -0.595386 \\ 8 & 1.284643 & 3.094911 & 0.517837 \\ 8 & -0.011031 & -1.799680 & 0.420545 \\ 8 & -0.215087 & 4.199968 & -1.786615 \\ 8 & -2.065692 & 2.955043 & 0.141395 \\ 8 & 2.162555 & 0.812337 & 2.471162 \\ 8 & 4.004671 & -1.806907 & -2.044467 \\ 8 & 3.371533 & -2.885383 & -2.334607 \\ 8 & 2.398463 & -3.144641 & -1.634719\end{array}$


Singlet bidentate (adsorption site \#2, isomer \#2)

$\begin{array}{rrrr}8 & -3.854943 & 1.002826 & -0.410937 \\ 22 & -3.723585 & -0.836293 & -0.913061 \\ 8 & -3.305241 & -1.994674 & 0.593079 \\ 22 & -1.728946 & -1.588659 & 1.221365 \\ 8 & -0.375656 & -1.953299 & 0.066387 \\ 22 & -0.271533 & -0.360751 & -1.005685 \\ 8 & -0.436197 & 1.529382 & -1.002736 \\ 22 & 0.125074 & 3.033375 & 0.105216 \\ 8 & -1.720129 & 3.022760 & 0.631022 \\ 22 & -2.185152 & 1.488038 & -0.092236 \\ 8 & -1.933365 & -0.523700 & -1.588573 \\ 8 & -1.503676 & 0.191247 & 1.053293 \\ 8 & -0.477877 & -2.000124 & 2.607047 \\ 22 & 1.000200 & -1.474285 & 1.765180 \\ 8 & 1.951302 & -0.256342 & 2.746059 \\ 22 & 2.310454 & 1.007650 & 1.464587 \\ 8 & 1.534735 & 2.543761 & 1.338306 \\ 8 & 2.322646 & -2.221302 & 0.644743 \\ 22 & 2.531630 & -0.934126 & -0.560983 \\ 8 & 3.531802 & 0.098697 & -2.860781 \\ 8 & 4.027466 & -1.001204 & -3.095455 \\ 8 & 3.982804 & -1.852933 & -2.147532 \\ 8 & 1.083368 & -0.012204 & 0.472881 \\ 8 & 3.506973 & 0.417531 & 0.195133 \\ 8 & 1.293307 & -0.832723 & -1.890592 \\ 8 & -4.805417 & -1.375875 & -1.988496 \\ 8 & 0.518207 & 4.297065 & -0.819900\end{array}$

\section{Singlet bidentate (adsorption site \#2, isomer \#3)}

$\begin{array}{rrrr}8 & -4.090726 & 0.337071 & -0.115063 \\ 22 & -3.659686 & -1.465592 & -0.582481 \\ 8 & -2.859941 & -2.445745 & 0.895628 \\ 22 & -1.331195 & -1.719210 & 1.319510 \\ 8 & -0.080051 & -1.886481 & 0.017692 \\ 22 & -0.399428 & -0.347778 & -1.094896 \\ 8 & -0.916485 & 1.473605 & -1.118266 \\ 22 & -0.546261 & 3.109820 & -0.125258 \\ 8 & -2.285963 & 2.773102 & 0.611118 \\ 22 & -2.522770 & 1.146391 & -0.015825 \\ 8 & -2.052512 & -0.844501 & -1.476316 \\ 8 & -1.479420 & 0.059778 & 1.074632 \\ 8 & 0.120973 & -1.811926 & 2.564679 \\ 22 & 1.373978 & -1.069125 & 1.543125 \\ 8 & 2.207015 & 0.356877 & 2.341744 \\ 22 & 2.140363 & 1.590122 & 0.982126 \\ 8 & 1.069346 & 2.939968 & 0.930388 \\ 8 & 2.653847 & -1.655056 & 0.301537 \\ 22 & 2.484608 & -0.397775 & -0.984007 \\ 8 & 4.103520 & -1.549746 & -2.169989 \\ 8 & 5.074947 & -1.862717 & -1.398867 \\ 8 & 5.150690 & -1.264564 & -0.333138 \\ 8 & 1.018759 & 0.298311 & 0.211819 \\ 8 & 3.243195 & 1.169155 & -0.437513 \\ 8 & 1.133939 & -0.546146 & -2.146807 \\ 8 & -4.730488 & -2.253596 & -1.503624 \\ 8 & -0.514569 & 4.383763 & -1.115960\end{array}$


Singlet monodentate (adsorption site \#2, isomer \#1)

$\begin{array}{rrrr}8 & -3.960940 & 0.251821 & -0.675787 \\ 22 & -2.412737 & 1.094796 & -0.543850 \\ 8 & -1.577147 & 0.363254 & 0.946340 \\ 22 & -1.504941 & -1.281643 & 1.677467 \\ 8 & -0.265284 & -1.008376 & 3.107831 \\ 22 & 1.141179 & -0.530014 & 2.125715 \\ 8 & 2.601050 & -1.375159 & 1.293519 \\ 22 & 2.649300 & -0.521786 & -0.273250 \\ 8 & 1.030452 & 0.430919 & 0.433304 \\ 22 & 2.037584 & 1.905110 & 1.013157 \\ 8 & 3.354994 & 1.142391 & -0.033071 \\ 22 & -3.500711 & -1.599807 & -0.563244 \\ 8 & -4.429508 & -2.632928 & -1.392626 \\ 8 & -1.760429 & -1.217774 & -1.327526 \\ 22 & -0.178916 & -0.590468 & -0.844637 \\ 8 & 1.482242 & -1.038121 & -1.554162 \\ 8 & -2.962653 & -2.130150 & 1.229798 \\ 8 & -0.659246 & 1.145703 & -1.440159 \\ 22 & -0.404383 & 2.997019 & -0.880974 \\ 8 & 1.019191 & 3.165332 & 0.422036 \\ 8 & -0.064926 & -1.758172 & 0.681047 \\ 8 & -0.193429 & 3.952444 & -2.165288 \\ 8 & -2.241760 & 2.835881 & -0.353544 \\ 8 & 1.846720 & 1.081525 & 2.641589 \\ 8 & 4.326829 & -1.655107 & -1.386068 \\ 8 & 3.824158 & -2.475534 & -2.236557 \\ 8 & 4.607154 & -3.129272 & -2.894234\end{array}$

\section{Singlet monodentate (adsorption site \#2, isomer}

$\begin{array}{rrrr}8 & -4.074112 & 0.659132 & -0.367934 \\ 22 & -3.780962 & -1.168563 & -0.845541 \\ 8 & -3.197127 & -2.251884 & 0.661658 \\ 22 & -1.644597 & -1.684062 & 1.219193 \\ 8 & -0.309644 & -1.939826 & 0.015804 \\ 22 & -0.397471 & -0.362219 & -1.081719 \\ 8 & -0.739425 & 1.502028 & -1.096271 \\ 22 & -0.289722 & 3.070236 & -0.028308 \\ 8 & -2.105989 & 2.891085 & 0.562613 \\ 22 & -2.447780 & 1.308251 & -0.125620 \\ 8 & -2.057164 & -0.691161 & -1.597233 \\ 8 & -1.604637 & 0.105277 & 1.014096 \\ 8 & -0.310249 & -1.947600 & 2.563639 \\ 22 & 1.081173 & -1.304123 & 1.657585 \\ 8 & 1.962280 & 0.015723 & 2.574894 \\ 22 & 2.134270 & 1.285017 & 1.259412 \\ 8 & 1.206550 & 2.736015 & 1.154248 \\ 8 & 2.415642 & -1.954506 & 0.499685 \\ 22 & 2.448249 & -0.671538 & -0.741351 \\ 8 & 4.347863 & -1.232856 & -1.933933 \\ 8 & 5.391115 & -0.869980 & -1.283464 \\ 8 & 6.466681 & -1.077333 & -1.809272 \\ 8 & 0.968204 & 0.133370 & 0.344967 \\ 8 & 3.324770 & 0.792358 & -0.058123 \\ 8 & 1.183930 & -0.693298 & -2.015279 \\ 8 & -4.846074 & -1.833012 & -1.865608 \\ 8 & -0.056307 & 4.355720 & -0.977029\end{array}$


Singlet bidentate (adsorption site \#3, isomer \#1)

$\begin{array}{rrrr}8 & 3.849417 & 1.142909 & -0.713833 \\ 22 & 2.130677 & 1.507167 & -0.527877 \\ 8 & 1.222214 & 0.001347 & -1.130680 \\ 22 & 1.561294 & -1.770808 & -1.059898 \\ 8 & -0.013614 & -2.452916 & -1.890976 \\ 22 & -1.191473 & -1.767586 & -0.738408 \\ 8 & -2.059482 & -2.362820 & 0.812491 \\ 22 & -1.908990 & -0.898228 & 1.840532 \\ 8 & -1.005751 & -0.123067 & 0.306143 \\ 22 & -2.624598 & 0.676547 & -0.373435 \\ 8 & -3.184633 & 0.295184 & 1.379759 \\ 22 & 4.066256 & -0.570217 & 0.109053 \\ 8 & 5.484984 & -0.862448 & 0.828409 \\ 8 & 2.562833 & -0.205682 & 1.278508 \\ 22 & 0.795036 & -0.181946 & 1.281249 \\ 8 & -0.370752 & -0.536279 & 2.697926 \\ 8 & 3.287068 & -2.004502 & -0.948170 \\ 8 & 0.800781 & 1.679444 & 0.914497 \\ 22 & -0.235400 & 2.929834 & -0.161531 \\ 8 & -1.948945 & 2.208267 & -0.731120 \\ 8 & 0.663967 & -1.938618 & 0.511412 \\ 8 & -0.388619 & 4.343172 & 0.600946 \\ 8 & 1.308339 & 2.862916 & -1.293491 \\ 8 & -2.455931 & -0.734908 & -1.561917 \\ 8 & -4.845509 & 0.685981 & -0.993220 \\ 8 & -5.366685 & -0.466765 & -0.808472 \\ 8 & -4.669886 & -1.324312 & -0.274843\end{array}$

\section{Singlet bidentate (adsorption site \#3, isomer \#2)}

$\begin{array}{rrrr}8 & 3.495472 & 1.917584 & -0.556404 \\ 22 & 4.096642 & 0.253168 & 0.169544 \\ 8 & 3.697869 & -1.235720 & -1.014596 \\ 22 & 1.968980 & -1.413555 & -1.170542 \\ 8 & 1.156985 & -1.913537 & 0.373133 \\ 22 & 0.808014 & -0.239002 & 1.254056 \\ 8 & 0.341207 & 1.576483 & 1.001284 \\ 22 & -0.930387 & 2.569783 & -0.079216 \\ 8 & 0.630942 & 3.013253 & -1.097667 \\ 22 & 1.739282 & 1.848780 & -0.379265 \\ 8 & 2.525190 & 0.199282 & 1.305885 \\ 8 & 1.219292 & 0.225494 & -1.125676 \\ 8 & 0.611592 & -2.421309 & -2.063021 \\ 22 & -0.687829 & -2.189879 & -0.868110 \\ 8 & -2.240065 & -1.533858 & -1.606575 \\ 22 & -2.706540 & -0.217537 & -0.444010 \\ 8 & -4.859534 & 0.206415 & -1.211001 \\ 8 & -5.288000 & 1.239568 & -0.590042 \\ 8 & -4.635784 & 1.597105 & 0.387097 \\ 8 & -1.323658 & -3.073876 & 0.657008 \\ 22 & -1.618840 & -1.666629 & 1.720858 \\ 8 & -0.235677 & -0.952293 & 2.624718 \\ 8 & -0.932911 & -0.603359 & 0.239399 \\ 8 & -3.169741 & -0.864989 & 1.246165 \\ 8 & -2.326479 & 1.447784 & -0.790691 \\ 8 & 5.530690 & 0.253928 & 0.917886\end{array}$


Singlet bidentate (adsorption site \#3, isomer \#3)

$\begin{array}{rrrr}8 & 3.481226 & 1.841866 & -0.664029 \\ 22 & 4.085886 & 0.120522 & -0.090173 \\ 8 & 3.531218 & -1.291699 & -1.305926 \\ 22 & 1.789897 & -1.410662 & -1.305268 \\ 8 & 1.098984 & -1.963185 & 0.277365 \\ 22 & 0.897660 & -0.325731 & 1.264183 \\ 8 & 0.490728 & 1.521408 & 1.159626 \\ 22 & -0.834152 & 2.649865 & 0.296984 \\ 8 & 0.619704 & 3.061733 & -0.878497 \\ 22 & 1.745322 & 1.817651 & -0.340134 \\ 8 & 2.628569 & 0.042112 & 1.187863 \\ 8 & 1.109912 & 0.250610 & -1.111053 \\ 8 & 0.325298 & -2.316337 & -2.130111 \\ 22 & -0.865340 & -2.099675 & -0.821172 \\ 8 & -2.424074 & -1.341214 & -1.399076 \\ 22 & -2.735591 & -0.053676 & -0.127230 \\ 8 & -5.012571 & 0.494918 & -0.324355 \\ 8 & -5.121675 & 1.122620 & -1.425722 \\ 8 & -4.144419 & 1.071637 & -2.174558 \\ 8 & -1.411843 & -3.026278 & 0.720067 \\ 22 & -1.553571 & -1.660804 & 1.861201 \\ 8 & -0.067613 & -1.067116 & 2.679943 \\ 8 & -0.936563 & -0.562041 & 0.378412 \\ 8 & -3.098979 & -0.758438 & 1.545938 \\ 8 & -2.348481 & 1.630117 & -0.319034 \\ 8 & 5.582799 & 0.038977 & 0.517221 \\ 8 & -1.260028 & 3.897212 & 1.235352\end{array}$

Singlet monodentate (adsorption site \#3, isomer

$\begin{array}{rrrr}8 & 3.449492 & 2.010872 & -0.625311 \\ 22 & 1.707238 & 1.888110 & -0.358238 \\ 8 & 1.191281 & 0.275569 & -1.127619 \\ 22 & 1.975554 & -1.343459 & -1.270730 \\ 8 & 0.595550 & -2.342868 & -2.131896 \\ 22 & -0.652469 & -2.171825 & -0.870946 \\ 8 & -1.204855 & -3.113372 & 0.658811 \\ 22 & -1.453937 & -1.750390 & 1.787452 \\ 8 & -0.851220 & -0.632051 & 0.312482 \\ 22 & -2.653131 & -0.236540 & -0.257722 \\ 8 & -3.033755 & -0.932890 & 1.425150 \\ 22 & 4.135118 & 0.337237 & -0.004235 \\ 8 & 5.612460 & 0.351803 & 0.654843 \\ 8 & 2.641487 & 0.189617 & 1.224848 \\ 22 & 0.934109 & -0.280837 & 1.251685 \\ 8 & -0.031406 & -1.063564 & 2.645734 \\ 8 & 3.706032 & -1.122032 & -1.214489 \\ 8 & 0.418573 & 1.535336 & 1.099013 \\ 22 & -0.944949 & 2.556210 & 0.160071 \\ 8 & -2.388853 & 1.452640 & -0.496339 \\ 8 & 1.261122 & -1.915562 & 0.295182 \\ 8 & -1.493808 & 3.773733 & 1.070136 \\ 8 & 0.530215 & 3.052268 & -0.958897 \\ 8 & -2.224864 & -1.497467 & -1.517969 \\ 8 & -4.909506 & -0.001307 & -0.781849\end{array}$




$\begin{array}{llll}8 & -5.242014 & 1.235209 & -0.750876 \\ 8 & -6.406647 & 1.498174 & -0.983631\end{array}$

Singlet monodentate (adsorption site \#3, isomer \#2)

$\begin{array}{rrrr}8 & 3.851180 & 1.254310 & -0.772936 \\ 22 & 4.131778 & -0.464816 & 0.016615 \\ 8 & 3.369921 & -1.899928 & -1.051384 \\ 22 & 1.635857 & -1.716485 & -1.119219 \\ 8 & 0.813554 & -1.950662 & 0.480884 \\ 22 & 0.885552 & -0.197905 & 1.271425 \\ 8 & 0.803453 & 1.664239 & 0.934780 \\ 22 & -0.287407 & 2.888716 & -0.115618 \\ 8 & 1.252834 & 2.906440 & -1.257693 \\ 22 & 2.129548 & 1.567033 & -0.526999 \\ 8 & 2.653906 & -0.144786 & 1.231997 \\ 8 & 1.252850 & 0.046257 & -1.142266 \\ 8 & 0.056626 & -2.446827 & -1.907295 \\ 22 & -1.108996 & -1.866948 & -0.690219 \\ 8 & -2.487702 & -0.915099 & -1.418686 \\ 22 & -2.596843 & 0.534195 & -0.285154 \\ 8 & -4.843040 & 0.751784 & -0.874719 \\ 8 & -5.390508 & -0.382548 & -0.664119 \\ 8 & -6.576129 & -0.484950 & -0.923825 \\ 8 & -1.862090 & -2.509652 & 0.907686 \\ 22 & -1.773219 & -1.028253 & 1.904907 \\ 8 & -0.227389 & -0.602233 & 2.717264 \\ 8 & -0.940509 & -0.218258 & 0.343179 \\ 8 & -3.133303 & 0.079957 & 1.443702 \\ 8 & -1.960437 & 2.084004 & -0.670165 \\ 8 & 5.576202 & -0.728972 & 0.694989 \\ 8 & -0.504162 & 4.279199 & 0.675325\end{array}$

Singlet bidentate (adsorption site \#4, isomer \#1)

$\begin{array}{rrrr}8 & 2.334620 & 2.759175 & -0.425921 \\ 22 & 0.646971 & 2.241071 & -0.474548 \\ 8 & 0.532166 & 0.515234 & -1.142743 \\ 22 & 1.698634 & -0.895214 & -1.076084 \\ 8 & 0.592303 & -2.018148 & -2.116471 \\ 22 & -0.819648 & -1.958146 & -1.006275 \\ 8 & -1.484134 & -3.037639 & 0.394424 \\ 22 & -2.059307 & -1.787104 & 1.521856 \\ 8 & -1.504808 & -0.509315 & 0.160587 \\ 22 & -3.215948 & -0.450806 & -0.642594 \\ 8 & -3.707688 & -1.278096 & 0.947702 \\ 22 & 3.299221 & 1.258109 & 0.247513 \\ 8 & 4.686056 & 1.506575 & 1.051385 \\ 8 & 1.785952 & 0.654075 & 1.290278 \\ 22 & 0.124436 & 0.047519 & 1.236202 \\ 8 & -0.850229 & -0.886879 & 2.527684 \\ 8 & 3.294156 & -0.140287 & -1.084368 \\ 8 & -0.588437 & 1.783836 & 0.954802 \\ 22 & -2.084322 & 2.548309 & -0.076189 \\ 8 & -3.320696 & 1.260804 & -0.817590 \\ 8 & 0.687346 & -1.555798 & 0.336823 \\ 8 & -2.813303 & 3.723047 & 0.754589 \\ 8 & -0.662393 & 3.177916 & -1.186940 \\ 8 & -2.409322 & -1.530887 & -1.862627\end{array}$




$\begin{array}{lllr}8 & 3.031108 & -2.839484 & -0.627825 \\ 8 & 3.654401 & -2.673570 & 0.462727 \\ 8 & 3.370299 & -1.670839 & 1.126316\end{array}$

Singlet bidentate (adsorption site \#4, isomer \#2)

$\begin{array}{rrrr}8 & 2.771042 & 2.297629 & -0.259294 \\ 22 & 3.253514 & 0.996647 & 1.050531 \\ 8 & 3.257481 & -0.809783 & 0.346033 \\ 22 & 1.685962 & -1.278975 & -0.277043 \\ 8 & 3.311930 & -3.050324 & -1.162363 \\ 8 & 3.494062 & -2.461087 & -2.240359 \\ 8 & 2.829233 & -1.406114 & -2.410656 \\ 22 & 1.047468 & 2.031397 & -0.561544 \\ 8 & 0.093126 & 2.800375 & -1.820981 \\ 22 & -1.644692 & 2.525007 & -1.042289 \\ 8 & -2.430783 & 3.903655 & -0.752224 \\ 8 & 0.860599 & 0.208772 & -0.857072 \\ 22 & -0.130493 & 0.391389 & 1.422737 \\ 8 & 0.446427 & -1.420857 & 1.086039 \\ 22 & -0.937209 & -2.193719 & -0.254788 \\ 8 & -2.275113 & -1.946235 & -1.521648 \\ 22 & -3.064518 & -0.419670 & -0.938628 \\ 8 & -2.817390 & 1.125812 & -1.673048 \\ 8 & -0.572962 & 2.006795 & 0.516092 \\ 8 & 0.566138 & -2.671475 & -1.052263 \\ 8 & -1.965862 & -2.660160 & 1.271555 \\ 22 & -2.578389 & -1.066398 & 1.741216 \\ 8 & -1.446865 & -0.015556 & 2.673858 \\ 8 & -1.565573 & -0.379828 & 0.206289 \\ 8 & -3.978606 & -0.619843 & 0.663516 \\ 8 & 4.460083 & 1.398392 & 2.052411 \\ 8 & 1.476015 & 0.989217 & 1.798590\end{array}$

\section{Singlet monodentate (adsorption site \#4, isomer \#1)}

$\begin{array}{rrrr}8 & 1.824377 & 3.173589 & -0.388615 \\ 22 & 0.230188 & 2.425364 & -0.494636 \\ 8 & 0.480586 & 0.708216 & -1.126725 \\ 22 & 1.790781 & -0.560652 & -1.097254 \\ 8 & 0.836682 & -1.877218 & -2.106321 \\ 22 & -0.503663 & -2.075323 & -0.952506 \\ 8 & -0.935714 & -3.180890 & 0.522690 \\ 22 & -1.739533 & -2.000926 & 1.579351 \\ 8 & -1.392228 & -0.704667 & 0.151777 \\ 22 & -3.098871 & -0.949836 & -0.624399 \\ 8 & -3.449797 & -1.783178 & 0.993413 \\ 22 & 2.984279 & 1.839871 & 0.341498 \\ 8 & 4.294458 & 2.359835 & 1.136468 \\ 8 & 1.606520 & 1.044662 & 1.442083 \\ 22 & 0.105214 & 0.173331 & 1.236225 \\ 8 & -0.709221 & -0.880818 & 2.545293 \\ 8 & 3.267104 & 0.354603 & -0.865934 \\ 8 & -0.910619 & 1.751890 & 0.908844 \\ 22 & -2.513261 & 2.224981 & -0.143936 \\ 8 & -3.476379 & 0.716205 & -0.869365 \\ 8 & 0.967504 & -1.321317 & 0.348881 \\ 8 & -3.457376 & 3.248166 & 0.670470 \\ 8 & -1.215352 & 3.101332 & -1.244187\end{array}$




$\begin{array}{rrrr}8 & -2.146891 & -1.962859 & -1.799106 \\ 8 & 3.284362 & -2.455137 & -0.796110 \\ 8 & 3.943040 & -2.202657 & 0.258345 \\ 8 & 4.737324 & -3.050985 & 0.646154\end{array}$

\section{Singlet monodentate (adsorption site \#4, isomer \#2)}

$\begin{array}{rrrr}8 & -1.869477 & -3.219220 & -0.426723 \\ 22 & -3.033365 & -1.959655 & 0.413312 \\ 8 & -3.375899 & -0.442822 & -0.745494 \\ 22 & -1.934455 & 0.519654 & -0.944054 \\ 8 & -3.413644 & 2.374502 & -0.439163 \\ 8 & -2.678992 & 3.371510 & -0.170977 \\ 8 & -3.231598 & 4.400588 & 0.202690 \\ 22 & -0.297398 & -2.430392 & -0.553071 \\ 8 & 1.129400 & -3.076114 & -1.361305 \\ 22 & 2.454395 & -2.268037 & -0.243962 \\ 8 & 3.404873 & -3.336782 & 0.501445 \\ 8 & -0.567782 & -0.685971 & -1.108122 \\ 22 & -0.124404 & -0.265996 & 1.282346 \\ 8 & -1.018169 & 1.246026 & 0.475707 \\ 22 & 0.382841 & 1.981458 & -0.896102 \\ 8 & 1.995621 & 1.892106 & -1.809808 \\ 22 & 3.012041 & 0.913458 & -0.664562 \\ 8 & 3.415203 & -0.741490 & -0.934440 \\ 8 & 0.874957 & -1.832319 & 0.858106 \\ 8 & -1.041782 & 1.884628 & -1.961070 \\ 8 & 0.862783 & 3.097080 & 0.548381 \\ 22 & 1.706533 & 1.922316 & 1.589752 \\ 8 & 0.727334 & 0.774285 & 2.582240 \\ 8 & 1.341336 & 0.627068 & 0.176823 \\ 8 & 3.399431 & 1.746438 & 0.950484 \\ 8 & -4.288964 & -2.556674 & 1.239924 \\ 8 & -1.621649 & -1.158055 & 1.466234\end{array}$

Singlet bidentate (adsorption site \#5, isomer \#1)

$\begin{array}{rrrr}8 & -3.254725 & 0.367852 & -0.550020 \\ 22 & -1.753350 & 1.103271 & 0.022171 \\ 8 & -0.783589 & -0.223751 & 0.866520 \\ 22 & -0.783534 & -2.013243 & 1.108926 \\ 8 & 0.644537 & -2.122884 & 2.383374 \\ 22 & 1.900939 & -1.371433 & 1.366354 \\ 8 & 3.164528 & -2.050613 & 0.166121 \\ 22 & 3.034417 & -0.845810 & -1.153998 \\ 8 & 1.663868 & -0.019891 & -0.035731 \\ 22 & 2.881882 & 1.209461 & 0.723311 \\ 8 & 3.963524 & 0.642564 & -0.662454 \\ 22 & -2.995106 & -1.500996 & -0.913849 \\ 8 & -4.207604 & -2.183754 & -1.734261 \\ 8 & -1.353567 & -1.294148 & -1.896907 \\ 22 & 0.158704 & -0.674337 & -1.334736 \\ 8 & 1.714831 & -0.941489 & -2.354810 \\ 8 & -2.377781 & -2.532723 & 0.619162 \\ 8 & -0.350878 & 1.161393 & -1.295350 \\ 22 & 0.339194 & 2.845589 & -0.537902 \\ 8 & 1.938251 & 2.638148 & 0.535744 \\ 8 & 0.466258 & -2.198534 & -0.173385 \\ 8 & 0.429039 & 4.031065 & -1.631602\end{array}$




$\begin{array}{rrrr}8 & -1.293263 & 2.786413 & 0.419765 \\ 8 & 2.782239 & 0.043902 & 2.138436 \\ 8 & -4.040263 & 2.449789 & 1.007629 \\ 8 & -3.884136 & 1.823726 & 2.054265 \\ 8 & -2.874919 & 1.053557 & 2.122741\end{array}$

\section{Singlet bidentate (adsorption site \#5, isomer \#2)}

$\begin{array}{rrrr}8 & -3.151060 & 1.299919 & -0.240761 \\ 22 & -3.490537 & -0.566459 & -0.663601 \\ 8 & -3.100427 & -1.834930 & 0.758917 \\ 22 & -1.386155 & -1.816022 & 1.105718 \\ 8 & -0.303680 & -2.265528 & -0.256280 \\ 22 & -0.314083 & -0.671146 & -1.369032 \\ 8 & -0.297279 & 1.239661 & -1.288184 \\ 22 & 0.832599 & 2.759059 & -0.770002 \\ 8 & -0.678754 & 3.192192 & 0.293034 \\ 22 & -1.491699 & 1.573079 & 0.175734 \\ 8 & -1.686243 & 1.961032 & 2.493135 \\ 8 & -0.513604 & 2.097145 & 2.961653 \\ 8 & 0.425546 & 1.839509 & 2.193882 \\ 8 & -0.939227 & -0.085840 & 0.853752 \\ 8 & 0.044885 & -2.210669 & 2.320212 \\ 22 & 1.373990 & -1.728451 & 1.232974 \\ 8 & 2.605203 & -0.539906 & 1.909929 \\ 22 & 2.918061 & 0.508329 & 0.429216 \\ 8 & 2.388852 & 2.132133 & 0.197071 \\ 8 & 2.357875 & -2.725415 & 0.007457 \\ 22 & 2.430783 & -1.566148 & -1.366069 \\ 8 & 1.060183 & -1.322566 & -2.485546 \\ 8 & 1.393499 & -0.402912 & -0.202496 \\ 8 & 3.737469 & -0.357155 & -0.980365 \\ 8 & -4.912123 & -0.818768 & -1.381356 \\ 8 & -1.975814 & -0.844191 & -1.813902 \\ 8 & 1.144067 & 3.792627 & -1.971234\end{array}$

\section{Singlet monodentate (adsorption site \#5, isomer \#1)}

$\begin{array}{rrrr}8 & 3.332143 & -0.761685 & -0.911944 \\ 22 & 2.027681 & 0.402421 & -0.666151 \\ 8 & 0.534390 & -0.684739 & -1.133974 \\ 22 & 0.066090 & -2.402030 & -0.910474 \\ 8 & -1.685002 & -2.516594 & -1.681754 \\ 22 & -2.525153 & -1.377547 & -0.613883 \\ 8 & -3.425938 & -1.362925 & 1.041761 \\ 22 & -2.718267 & 0.103314 & 1.770439 \\ 8 & -1.642229 & 0.186229 & 0.133293 \\ 22 & -2.788122 & 1.442559 & -0.687469 \\ 8 & -3.458062 & 1.600225 & 1.026275 \\ 22 & 2.834943 & -2.215498 & 0.220212 \\ 8 & 4.030812 & -2.954381 & 1.021279 \\ 8 & 1.715014 & -1.003342 & 1.263893 \\ 22 & 0.051102 & -0.373204 & 1.168422 \\ 8 & -1.135466 & -0.027877 & 2.585777 \\ 8 & 1.523083 & -3.332159 & -0.665380 \\ 8 & 0.660516 & 1.289708 & 0.572086 \\ 22 & 0.279882 & 2.588743 & -0.784449 \\ 8 & -1.584576 & 2.507376 & -1.315054 \\ 8 & -0.760321 & -2.045077 & 0.665640\end{array}$




$\begin{array}{rrrr}8 & 0.739779 & 4.028316 & -0.193985 \\ 8 & 1.735114 & 1.780922 & -1.725464 \\ 8 & -3.383744 & -0.058150 & -1.555434 \\ 8 & 3.335846 & 1.707180 & 0.750676 \\ 8 & 3.291966 & 2.931590 & 0.380168 \\ 8 & 3.799246 & 3.751296 & 1.126369\end{array}$

Singlet monodentate (adsorption site \#5, isomer \#2)

$\begin{array}{rrrr}8 & 3.356500 & -0.571031 & -1.020928 \\ 22 & 2.990671 & -1.993048 & 0.196039 \\ 8 & 1.732210 & -3.239901 & -0.591352 \\ 22 & 0.211474 & -2.421871 & -0.842171 \\ 8 & -0.591049 & -2.044990 & 0.741660 \\ 22 & 0.123834 & -0.303076 & 1.136574 \\ 8 & 0.612477 & 1.370115 & 0.453839 \\ 22 & 0.094581 & 2.577203 & -0.947466 \\ 8 & 1.561763 & 1.818500 & -1.906335 \\ 22 & 1.991272 & 0.523726 & -0.789588 \\ 8 & 3.365341 & 1.926770 & 0.459552 \\ 8 & 2.709413 & 2.884998 & 0.995585 \\ 8 & 3.357580 & 3.731784 & 1.586168 \\ 8 & 0.563328 & -0.689500 & -1.148754 \\ 8 & 1.828204 & -0.809902 & 1.227544 \\ 8 & -1.550923 & -2.681406 & -1.553681 \\ 22 & -2.432988 & -1.549688 & -0.513394 \\ 8 & -3.404370 & -0.335340 & -1.485097 \\ 22 & -2.886942 & 1.239959 & -0.701953 \\ 8 & -1.777932 & 2.353415 & -1.411388 \\ 8 & -3.281636 & -1.516135 & 1.169878 \\ 22 & -2.649976 & 0.024412 & 1.808669 \\ 8 & -1.037392 & 0.037782 & 2.575682 \\ 8 & -1.632976 & 0.101138 & 0.133247 \\ 8 & -3.511333 & 1.432757 & 1.025922 \\ 8 & 4.257013 & -2.609007 & 0.992655 \\ 8 & 0.478488 & 4.071509 & -0.447647\end{array}$

Singlet monodentate (adsorption site \#5, isomer

$\begin{array}{rrrr}8 & 3.322985 & -0.213714 & -1.242149 \\ 22 & 3.150882 & -1.625816 & 0.024068 \\ 8 & 1.984261 & -3.006631 & -0.675330 \\ 22 & 0.382132 & -2.341365 & -0.864687 \\ 8 & -0.364693 & -2.009100 & 0.754695 \\ 22 & 0.189713 & -0.195003 & 1.077956 \\ 8 & 0.479151 & 1.499573 & 0.345936 \\ 22 & -0.214014 & 2.632534 & -1.047716 \\ 8 & 1.300256 & 2.009072 & -2.050418 \\ 22 & 1.872036 & 0.760606 & -0.955194 \\ 8 & 3.206876 & 2.086298 & 0.409333 \\ 8 & 2.767615 & 2.099379 & 1.603567 \\ 8 & 3.427497 & 2.709125 & 2.432086 \\ 8 & 0.554179 & -0.589013 & -1.219813 \\ 8 & 1.944322 & -0.528742 & 1.096177 \\ 8 & -1.378521 & -2.789672 & -1.472824 \\ 22 & -2.316571 & -1.728190 & -0.406830 \\ 8 & -3.444955 & -0.639783 & -1.353327 \\ 22 & -3.035324 & 1.000934 & -0.639790 \\ 8 & -2.065513 & 2.180790 & -1.434086\end{array}$




$\begin{array}{rrrr}8 & -3.077155 & -1.726228 & 1.319236 \\ 22 & -2.564261 & -0.115432 & 1.885748 \\ 8 & -0.921643 & 0.069198 & 2.567132 \\ 8 & -1.642970 & 0.004657 & 0.158930 \\ 8 & -3.588395 & 1.184032 & 1.115458 \\ 8 & 4.509218 & -2.078810 & 0.779845 \\ 8 & -0.040146 & 4.171837 & -0.586727\end{array}$

Singlet monodentate (adsorption site \#5, isomer \#4)

$\begin{array}{rrrr}8 & 3.260088 & -0.013033 & -1.237291 \\ 22 & 3.119832 & -1.523481 & -0.093889 \\ 8 & 1.977588 & -2.872615 & -0.885735 \\ 22 & 0.361648 & -2.220556 & -1.009725 \\ 8 & -0.384925 & -2.024638 & 0.630431 \\ 22 & 0.140598 & -0.225382 & 1.090115 \\ 8 & 0.399436 & 1.520421 & 0.483950 \\ 22 & -0.330223 & 2.745953 & -0.819185 \\ 8 & 1.196887 & 2.230060 & -1.868624 \\ 22 & 1.786514 & 0.907602 & -0.878407 \\ 8 & 3.058635 & 2.099431 & 0.671230 \\ 8 & 3.922415 & 1.302907 & 1.168768 \\ 8 & 4.600495 & 1.722488 & 2.091745 \\ 8 & 0.497473 & -0.442977 & -1.239328 \\ 8 & 1.895900 & -0.551310 & 1.067860 \\ 8 & -1.390265 & -2.657417 & -1.644224 \\ 22 & -2.342965 & -1.699367 & -0.494165 \\ 8 & -3.498870 & -0.567648 & -1.352023 \\ 22 & -3.114426 & 1.025685 & -0.526204 \\ 8 & -2.167781 & 2.274413 & -1.238626 \\ 8 & -3.096478 & -1.837919 & 1.229639 \\ 22 & -2.612005 & -0.262512 & 1.910094 \\ 8 & -0.970401 & -0.102162 & 2.597954 \\ 8 & -1.699899 & 0.000927 & 0.194087 \\ 8 & -3.661753 & 1.072284 & 1.238796 \\ 8 & 4.500246 & -1.972106 & 0.627940 \\ 8 & -0.213466 & 4.262051 & -0.277793\end{array}$

Singlet bidentate (adsorption site \#6, isomer \#1)

$\begin{array}{rrrr}8 & 3.054731 & 1.720724 & -0.951232 \\ 22 & 1.299061 & 1.646856 & -0.708433 \\ 8 & 0.518425 & 0.228911 & -1.626689 \\ 22 & 1.351890 & -1.368377 & -1.410952 \\ 8 & -0.037902 & -2.253744 & -2.366137 \\ 22 & -1.323299 & -1.690491 & -1.261717 \\ 8 & -2.297927 & -2.640352 & 0.053446 \\ 22 & -2.451451 & -1.405871 & 1.315517 \\ 8 & -1.516960 & -0.220515 & 0.094497 \\ 22 & -3.126819 & 0.445734 & -0.656407 \\ 8 & -3.859782 & -0.359334 & 0.853013 \\ 22 & 3.673315 & 0.013746 & -0.389560 \\ 8 & 5.008256 & -0.141860 & 0.526904 \\ 8 & 2.005055 & -0.200846 & 0.532982 \\ 22 & 0.207685 & -0.330556 & 1.126767 \\ 8 & -1.014799 & -1.031556 & 2.365603 \\ 8 & 3.093076 & -1.365771 & -1.625693 \\ 8 & 0.125197 & 1.510088 & 0.892602 \\ 22 & -1.094433 & 2.844790 & 0.109077 \\ 8 & -2.717385 & 2.121002 & -0.640539\end{array}$




$\begin{array}{rrrr}8 & 0.229548 & -1.871163 & 0.040112 \\ 8 & -1.322921 & 4.099071 & 1.101061 \\ 8 & 0.335818 & 3.069290 & -1.110019 \\ 8 & -2.705306 & -0.684013 & -2.005882 \\ 8 & 1.437873 & 0.240172 & 3.106860 \\ 8 & 2.005732 & -0.858688 & 3.338667 \\ 8 & 1.685411 & -1.789947 & 2.578642\end{array}$

Singlet monodentate (adsorption site \#6, isomer \#1)

$\begin{array}{rrrr}8 & 3.094452 & 0.648863 & -1.558181 \\ 22 & 1.352296 & 0.878820 & -1.320381 \\ 8 & 0.351979 & -0.674916 & -1.535277 \\ 22 & 1.053083 & -2.124647 & -0.674447 \\ 8 & -0.491978 & -3.119062 & -1.186574 \\ 22 & -1.616041 & -1.947004 & -0.444404 \\ 8 & -2.640052 & -2.171764 & 1.133498 \\ 22 & -2.551681 & -0.549611 & 1.830025 \\ 8 & -1.564741 & -0.045901 & 0.233918 \\ 22 & -3.134126 & 0.467012 & -0.700153 \\ 8 & -3.856503 & 0.413058 & 1.014623 \\ 22 & 3.560764 & -0.744287 & -0.354613 \\ 8 & 4.907667 & -0.625879 & 0.549440 \\ 8 & 1.927456 & -0.392373 & 0.586093 \\ 22 & 0.189029 & 0.014405 & 1.198291 \\ 8 & -1.003113 & -0.006287 & 2.628941 \\ 8 & 2.765596 & -2.428955 & -0.912162 \\ 8 & 0.340432 & 1.598794 & 0.215418 \\ 22 & -0.780586 & 2.657871 & -1.013057 \\ 8 & -2.542354 & 1.954745 & -1.347061 \\ 8 & -0.051878 & -1.828063 & 0.851556 \\ 8 & -0.740197 & 4.225878 & -0.622941 \\ 8 & 0.539338 & 2.128714 & -2.262745 \\ 8 & -2.918114 & -1.133684 & -1.511241 \\ 8 & 1.483590 & 1.136572 & 2.787680 \\ 8 & 2.454744 & 1.690511 & 2.167382 \\ 8 & 3.243647 & 2.335210 & 2.834162\end{array}$

\section{Singlet monodentate (adsorption site \#6, isomer \#2)}

$\begin{array}{rrrr}8 & 3.258159 & 0.606526 & -1.471113 \\ 22 & 3.718741 & -0.521216 & -0.008815 \\ 8 & 3.071613 & -2.325788 & -0.311766 \\ 22 & 1.330568 & -2.133903 & -0.259359 \\ 8 & 0.119039 & -1.682605 & 1.133618 \\ 22 & 0.200169 & 0.212552 & 1.137079 \\ 8 & 1.262265 & 1.811269 & 2.503362 \\ 8 & 0.523867 & 2.847458 & 2.481620 \\ 8 & 0.935068 & 3.842551 & 3.060313 \\ 22 & 1.496363 & 0.753200 & -1.390643 \\ 8 & 0.672974 & 1.732205 & -2.606668 \\ 22 & -0.822408 & 2.301043 & -1.592542 \\ 8 & -0.977348 & 3.905696 & -1.463131 \\ 8 & 0.631178 & -0.892191 & -1.386759 \\ 8 & 1.998736 & -0.103878 & 0.769648 \\ 8 & 0.250715 & 1.607480 & -0.100723 \\ 8 & -0.089766 & -3.337043 & -0.666216 \\ 22 & -1.363157 & -2.193347 & -0.158426 \\ 8 & -2.646049 & -1.713890 & -1.430379\end{array}$




$\begin{array}{rrrr}22 & -3.015291 & -0.004702 & -0.974210 \\ 8 & -2.478904 & 1.360991 & -1.885002 \\ 8 & -2.453861 & -2.192156 & 1.387976 \\ 22 & -2.532489 & -0.457241 & 1.735938 \\ 8 & -1.095193 & 0.375682 & 2.482648 \\ 8 & -1.483782 & -0.206940 & 0.120895 \\ 8 & -3.843814 & 0.220211 & 0.680383 \\ 8 & 5.060740 & -0.235643 & 0.856480\end{array}$

Triplet bidentate (adsorption site \#1, isomer \#1)

$\begin{array}{rrrr}8 & 3.900767 & -0.075248 & -0.664569 \\ 22 & 2.447227 & 0.925880 & -0.598400 \\ 8 & 1.045047 & -0.199987 & -1.094234 \\ 22 & 0.673795 & -1.925761 & -0.792409 \\ 8 & -1.043092 & -2.060873 & -1.676523 \\ 22 & -2.039288 & -1.063269 & -0.418810 \\ 8 & -2.904702 & -0.904817 & 1.266576 \\ 22 & -2.209641 & 0.583672 & 1.896422 \\ 8 & -1.140138 & 0.580822 & 0.258020 \\ 22 & -2.293007 & 1.779850 & -0.624698 \\ 8 & -2.970357 & 2.028367 & 1.067065 \\ 22 & 3.445819 & -1.623991 & 0.358366 \\ 8 & 4.636064 & -2.329396 & 1.194292 \\ 8 & 2.186292 & -0.553868 & 1.378601 \\ 22 & 0.541110 & 0.095057 & 1.297597 \\ 8 & -0.612040 & 0.440839 & 2.712136 \\ 8 & 2.180684 & -2.769847 & -0.573206 \\ 8 & 1.183618 & 1.759201 & 0.685955 \\ 22 & 0.709200 & 3.103391 & -0.647348 \\ 8 & -1.110333 & 2.855850 & -1.264168 \\ 8 & -0.218002 & -1.609268 & 0.750955 \\ 8 & 0.983996 & 4.589292 & -0.082471 \\ 8 & 2.207538 & 2.357526 & -1.587534 \\ 8 & -2.863754 & 0.218324 & -1.413392 \\ 8 & -2.005050 & -3.363323 & -0.182165 \\ 8 & -3.182428 & -3.619653 & -0.917311 \\ 8 & -3.780950 & -2.499723 & -1.152507\end{array}$

Triplet bidentate (adsorption site \#1, isomer \#2)

$\begin{array}{rrrr}8 & 4.033183 & 0.056662 & -0.580481 \\ 22 & 3.607398 & -1.582272 & 0.309808 \\ 8 & 2.423003 & -2.718835 & -0.726811 \\ 22 & 0.882341 & -1.923989 & -0.929935 \\ 8 & -0.068442 & -1.764457 & 0.609410 \\ 22 & 0.623616 & -0.057131 & 1.257054 \\ 8 & 1.197784 & 1.677953 & 0.795802 \\ 22 & 0.676392 & 3.065723 & -0.477561 \\ 8 & 2.243644 & 2.467004 & -1.412910 \\ 22 & 2.541490 & 0.997840 & -0.497561 \\ 8 & 2.281866 & -0.643061 & 1.383091 \\ 8 & 1.203945 & -0.160587 & -1.073503 \\ 8 & -0.781285 & -2.076387 & -1.833684 \\ 22 & -1.857519 & -1.260531 & -0.700883 \\ 8 & -3.404036 & -2.505898 & -1.059794 \\ 8 & -4.603229 & -2.108610 & -0.624209 \\ 8 & -4.637295 & -1.254028 & 0.364662 \\ 8 & -2.713714 & -1.328579 & 1.142791\end{array}$




$\begin{array}{rrrr}22 & -2.140489 & 0.248060 & 1.812095 \\ 8 & -0.583514 & 0.183132 & 2.672739 \\ 8 & -1.080707 & 0.399311 & 0.215269 \\ 22 & -2.263265 & 1.600826 & -0.616022 \\ 8 & -2.740011 & 0.118546 & -1.551260 \\ 8 & -3.004770 & 1.667424 & 1.091578 \\ 8 & -1.114271 & 2.768737 & -1.151018 \\ 8 & 4.804518 & -2.299555 & 1.125197 \\ 8 & 0.850934 & 4.527781 & 0.181392\end{array}$

\section{Triplet bidentate (adsorption site \#1, isomer \#3)}

$\begin{array}{rrrr}8 & 3.670328 & 1.194728 & -0.493509 \\ 22 & 3.681455 & -0.461421 & 0.460369 \\ 8 & 2.882252 & -1.906814 & -0.558768 \\ 22 & 1.184132 & -1.622994 & -0.805827 \\ 8 & 0.172650 & -1.655562 & 0.694541 \\ 22 & 0.368986 & 0.213257 & 1.280498 \\ 8 & 0.476976 & 2.021475 & 0.733899 \\ 22 & -0.383404 & 3.144932 & -0.617514 \\ 8 & 1.306886 & 2.958533 & -1.505989 \\ 22 & 1.971275 & 1.673747 & -0.507979 \\ 8 & 2.116432 & 0.094362 & 1.468103 \\ 8 & 1.031485 & 0.154970 & -1.029859 \\ 8 & -0.432336 & -2.020510 & -1.863014 \\ 22 & -1.707576 & -1.652514 & -0.486869 \\ 8 & -1.695052 & -3.691359 & -0.292330 \\ 8 & -0.601690 & -4.404748 & -0.480175 \\ 8 & 0.357586 & -3.876551 & -1.215857 \\ 8 & -2.642852 & -1.717156 & 1.194303 \\ 22 & -2.422689 & -0.101018 & 1.810515 \\ 8 & -0.867877 & 0.193040 & 2.661702 \\ 8 & -1.358660 & 0.193095 & 0.182692 \\ 22 & -2.810121 & 0.972214 & -0.730589 \\ 8 & -2.907484 & -0.732980 & -1.471836 \\ 8 & -3.557555 & 1.054504 & 0.931888 \\ 8 & -1.993715 & 2.351555 & -1.355218 \\ 8 & 5.003970 & -0.794881 & 1.327557 \\ 8 & -0.636996 & 4.627239 & -0.035293\end{array}$

Triplet monodentate (adsorption site \#1, isomer \#1)

$\begin{array}{rrrr}8 & 3.932266 & -0.416550 & -0.720647 \\ 22 & 2.568623 & 0.853554 & -0.748903 \\ 8 & 1.062313 & -0.146899 & -1.096273 \\ 22 & 0.561390 & -1.816196 & -0.648042 \\ 8 & -1.030214 & -2.071585 & -1.381670 \\ 22 & -2.113723 & -1.173001 & -0.105105 \\ 8 & -2.664516 & -0.268892 & 1.571571 \\ 22 & -1.934677 & 1.240705 & 1.855209 \\ 8 & -0.817468 & 1.115391 & 0.269340 \\ 22 & -2.223084 & 1.770500 & -0.834208 \\ 8 & -2.843821 & 2.418586 & 0.782931 \\ 22 & 3.338873 & -1.745131 & 0.333634 \\ 8 & 4.654085 & -2.650756 & 1.258645 \\ 8 & 2.203459 & -0.736824 & 1.345127 \\ 22 & 0.651171 & 0.283275 & 1.319273 \\ 8 & -0.286478 & 0.898581 & 2.706569 \\ 8 & 2.112164 & -2.789077 & -0.502055\end{array}$




$\begin{array}{rrrr}8 & 1.648589 & 1.721106 & 0.629479 \\ 22 & 0.563651 & 2.720997 & -0.790448 \\ 8 & -1.070758 & 2.618489 & -1.819842 \\ 8 & -0.336652 & -1.333194 & 0.877028 \\ 8 & 0.589563 & 4.211846 & -0.189388 \\ 8 & 2.205913 & 2.189950 & -1.786546 \\ 8 & -2.806806 & 0.119907 & -1.214169 \\ 8 & -3.269555 & -2.531756 & 0.119235 \\ 8 & -3.093054 & -3.764606 & -0.933851 \\ 8 & -4.072649 & -4.454155 & -0.964365\end{array}$

Triplet monodentate (adsorption site \#1, isomer

$\begin{array}{rrrr}8 & 4.019979 & -0.344328 & -0.714598 \\ 22 & 3.454929 & -1.774335 & 0.223114 \\ 8 & 2.284677 & -2.784343 & -0.705034 \\ 22 & 0.677353 & -1.887589 & -0.879137 \\ 8 & -0.122015 & -1.673117 & 0.706487 \\ 22 & 0.666913 & -0.010270 & 1.301361 \\ 8 & 1.489160 & 1.633035 & 0.763932 \\ 22 & 0.836823 & 2.976742 & -0.540131 \\ 8 & 2.377926 & 2.284492 & -1.524402 \\ 22 & 2.606513 & 0.848200 & -0.583103 \\ 8 & 2.353476 & -0.776321 & 1.302983 \\ 8 & 1.183085 & -0.176256 & -1.111775 \\ 8 & -0.902336 & -2.003702 & -1.713319 \\ 22 & -2.095543 & -1.241087 & -0.509707 \\ 8 & -3.358909 & -2.514051 & -0.724952 \\ 8 & -4.753865 & -2.072092 & -0.067451 \\ 8 & -5.598199 & -1.869439 & -0.906534 \\ 8 & -2.717323 & -0.861129 & 1.339716 \\ 22 & -2.005433 & 0.590560 & 1.890198 \\ 8 & -0.360623 & 0.327172 & 2.732359 \\ 8 & -0.895648 & 0.651890 & 0.309754 \\ 22 & -2.098387 & 1.727840 & -0.618442 \\ 8 & -2.769490 & 0.225673 & -1.380780 \\ 8 & -2.820589 & 2.004451 & 1.048377 \\ 8 & -0.918016 & 2.833136 & -1.279524 \\ 8 & 4.784429 & -2.715790 & 1.086901 \\ 8 & 1.105569 & 4.448048 & 0.056437\end{array}$

\section{Triplet bidentate (adsorption site \#2, isomer \#1)}

$\begin{array}{rrrr}8 & -3.844833 & 0.728980 & -0.332695 \\ 22 & -2.214416 & 1.394195 & -0.198172 \\ 8 & -1.330085 & 0.367112 & 1.076801 \\ 22 & -1.450777 & -1.357162 & 1.630601 \\ 8 & -0.137010 & -1.295360 & 2.992972 \\ 22 & 1.219503 & -0.754890 & 1.954345 \\ 8 & 2.572046 & -1.693812 & 1.115773 \\ 22 & 2.726802 & -0.849652 & -0.462437 \\ 8 & 1.199653 & 0.277996 & 0.277578 \\ 22 & 2.374511 & 1.590544 & 0.926929 \\ 8 & 3.608241 & 0.687291 & -0.126992 \\ 22 & -3.635039 & -1.166473 & -0.439849 \\ 8 & -4.752582 & -1.973618 & -1.283342 \\ 8 & -1.898787 & -0.982212 & -1.296413 \\ 22 & -0.257928 & -0.514665 & -0.898803 \\ 8 & 1.341893 & -1.015814 & -1.777103\end{array}$




$\begin{array}{rrrr}8 & -3.044570 & -1.951318 & 1.236821 \\ 8 & -0.627663 & 1.307353 & -1.316415 \\ 22 & -0.057674 & 3.080676 & -0.691533 \\ 8 & 1.526769 & 3.001465 & 0.406907 \\ 8 & -0.116148 & -1.829925 & 0.487206 \\ 8 & 0.067592 & 4.107742 & -1.927749 \\ 8 & -1.804555 & 3.074156 & 0.110232 \\ 8 & 2.065406 & 0.771200 & 2.526049 \\ 8 & 3.710536 & -2.047583 & -1.662660 \\ 8 & 3.131432 & -2.825340 & -2.619183 \\ 8 & 1.893963 & -2.620387 & -2.895761\end{array}$

Triplet bidentate (adsorption site \#2, isomer \#2)

$\begin{array}{rrrr}8 & 3.990447 & 0.662510 & 0.442344 \\ 22 & 3.633940 & -1.051605 & 0.848199 \\ 8 & 3.263145 & -2.061273 & -0.623904 \\ 22 & 1.651448 & -1.560199 & -1.332356 \\ 8 & 0.377387 & -1.938198 & -0.139442 \\ 22 & 0.223351 & -0.391304 & 0.960122 \\ 8 & 0.621558 & 1.482210 & 1.088877 \\ 22 & 0.073414 & 3.072037 & 0.068087 \\ 8 & 1.939335 & 2.943078 & -0.500860 \\ 22 & 2.299977 & 1.384835 & 0.167485 \\ 8 & 1.982039 & -0.664747 & 1.526849 \\ 8 & 1.658330 & 0.211656 & -1.085212 \\ 8 & 0.411672 & -1.783954 & -2.682343 \\ 22 & -1.105593 & -1.252856 & -1.796299 \\ 8 & -2.057055 & 0.011916 & -2.673746 \\ 22 & -2.215259 & 1.231330 & -1.284560 \\ 8 & -1.421624 & 2.774359 & -1.085227 \\ 8 & -2.351224 & -2.024587 & -0.695944 \\ 22 & -2.720464 & -0.930334 & 0.687535 \\ 8 & -3.945798 & -0.182046 & 2.691948 \\ 8 & -4.584744 & -1.287968 & 2.578270 \\ 8 & -4.110836 & -2.004709 & 1.450430 \\ 8 & -0.873756 & 0.173741 & -0.553980 \\ 8 & -3.370946 & 0.620620 & -0.045308 \\ 8 & -1.261352 & -0.818024 & 1.828518 \\ 8 & 4.821551 & -1.808847 & 2.036676 \\ 8 & -0.150367 & 4.314024 & 1.066970\end{array}$

Triplet bidentate (adsorption site \#2, isomer \#3)

$\begin{array}{rrrr}8 & -4.075707 & 0.424330 & 0.017705 \\ 22 & -3.710848 & -1.363982 & -0.552300 \\ 8 & -2.872939 & -2.452105 & 0.828845 \\ 22 & -1.310696 & -1.786000 & 1.216783 \\ 8 & -0.075867 & -1.898052 & -0.116523 \\ 22 & -0.442867 & -0.277619 & -1.123156 \\ 8 & -0.934046 & 1.543712 & -1.037832 \\ 22 & -0.466046 & 3.114393 & 0.028556 \\ 8 & -2.172511 & 2.766009 & 0.839355 \\ 22 & -2.489579 & 1.194690 & 0.118835 \\ 8 & -2.103706 & -0.758305 & -1.462836 \\ 8 & -1.430697 & 0.006155 & 1.080738 \\ 8 & 0.154407 & -1.912894 & 2.444084 \\ 22 & 1.396619 & -1.155241 & 1.432332 \\ 8 & 2.352250 & 0.175590 & 2.217550\end{array}$




$\begin{array}{rrrr}22 & 2.242906 & 1.475551 & 0.902186 \\ 8 & 1.210380 & 2.853533 & 0.964209 \\ 8 & 2.579635 & -1.824965 & 0.094055 \\ 22 & 2.447423 & -0.435370 & -1.206082 \\ 8 & 4.050688 & -1.253668 & -2.031843 \\ 8 & 5.044124 & -1.484299 & -1.131093 \\ 8 & 4.679925 & -1.582356 & 0.108694 \\ 8 & 1.036904 & 0.248834 & 0.162786 \\ 8 & 3.237430 & 1.088193 & -0.586029 \\ 8 & 1.036992 & -0.417666 & -2.273505 \\ 8 & -4.829346 & -2.071117 & -1.478970 \\ 8 & -0.471923 & 4.441410 & -0.886561\end{array}$

Triplet monodentate (adsorption site \#2, isomer

$\begin{array}{rrrr}8 & -4.078346 & -0.140188 & -0.250308 \\ 22 & -2.580614 & 0.956319 & -0.176183 \\ 8 & -1.621616 & 0.088069 & 1.118905 \\ 22 & -1.193594 & -1.603173 & 1.517890 \\ 8 & 0.137148 & -1.407728 & 2.781914 \\ 22 & 1.446213 & -0.649667 & 1.737618 \\ 8 & 2.749666 & -1.286607 & 0.637073 \\ 22 & 2.793260 & -0.273424 & -0.877284 \\ 8 & 0.816997 & 0.545442 & 0.396639 \\ 22 & 1.932978 & 1.944162 & 0.886978 \\ 8 & 3.096546 & 1.474070 & -0.414255 \\ 22 & -3.365358 & -1.767615 & -0.530365 \\ 8 & -4.418995 & -2.890441 & -1.544959 \\ 8 & -1.891508 & -1.101796 & -1.375892 \\ 22 & -0.205130 & -0.392219 & -0.977816 \\ 8 & 1.274847 & -0.566183 & -1.928978 \\ 8 & -2.692280 & -2.515400 & 0.989669 \\ 8 & -1.024906 & 1.325670 & -1.235216 \\ 22 & -0.795502 & 3.094231 & -0.408090 \\ 8 & 0.800085 & 3.249106 & 0.633128 \\ 8 & 0.053832 & -1.817457 & 0.260577 \\ 8 & -0.916743 & 4.243192 & -1.529777 \\ 8 & -2.544309 & 2.618192 & 0.320807 \\ 8 & 2.157632 & 0.888651 & 2.391809 \\ 8 & 4.231772 & -0.882326 & -1.786097 \\ 8 & 4.265947 & -2.496333 & -1.764601 \\ 8 & 5.015538 & -2.926617 & -0.925493\end{array}$

Triplet monodentate (adsorption site \#2, isomer \#2)

$\begin{array}{rrrr}8 & -4.129520 & 0.039738 & -0.169910 \\ 22 & -3.513803 & -1.593229 & -0.604481 \\ 8 & -2.839036 & -2.501806 & 0.823037 \\ 22 & -1.277459 & -1.716085 & 1.375761 \\ 8 & -0.074375 & -1.881140 & 0.068545 \\ 22 & -0.302808 & -0.346714 & -1.038022 \\ 8 & -1.036704 & 1.423037 & -1.131713 \\ 22 & -0.690360 & 3.112076 & -0.186045 \\ 8 & -2.434822 & 2.662356 & 0.574139 \\ 22 & -2.575307 & 1.050413 & -0.050511 \\ 8 & -2.027719 & -0.937266 & -1.441152 \\ 8 & -1.627939 & 0.023508 & 1.130826 \\ 8 & 0.083786 & -1.688505 & 2.617044 \\ 22 & 1.406303 & -0.898325 & 1.609063\end{array}$




$\begin{array}{rrrr}8 & 2.199315 & 0.547353 & 2.363329 \\ 22 & 2.010455 & 1.720206 & 0.938421 \\ 8 & 0.944266 & 3.099580 & 0.797702 \\ 8 & 2.669211 & -1.496088 & 0.445116 \\ 22 & 2.684958 & -0.405433 & -1.017592 \\ 8 & 4.059042 & -1.015622 & -2.019929 \\ 8 & 5.343755 & -1.310738 & -1.090558 \\ 8 & 5.452721 & -2.479677 & -0.819116 \\ 8 & 0.808667 & 0.418540 & 0.371827 \\ 8 & 3.123227 & 1.280273 & -0.404657 \\ 8 & 1.146582 & -0.503812 & -2.053028 \\ 8 & -4.656647 & -2.567529 & -1.673387 \\ 8 & -0.794255 & 4.349799 & -1.211246\end{array}$

Triplet bidentate (adsorption site \#3, isomer \#1)

$\begin{array}{rrrr}8 & 3.775883 & 1.135965 & -0.872393 \\ 22 & 2.059182 & 1.483283 & -0.653106 \\ 8 & 1.152354 & -0.066174 & -1.144797 \\ 22 & 1.528845 & -1.827090 & -0.998708 \\ 8 & -0.075632 & -2.572922 & -1.728824 \\ 22 & -1.202584 & -1.836163 & -0.565396 \\ 8 & -2.017716 & -2.389703 & 0.989950 \\ 22 & -1.846346 & -0.871599 & 1.976691 \\ 8 & -1.027327 & -0.144396 & 0.371330 \\ 22 & -2.720142 & 0.708335 & -0.207526 \\ 8 & -3.145332 & 0.287780 & 1.564178 \\ 22 & 4.052532 & -0.535105 & 0.016809 \\ 8 & 5.498014 & -0.768714 & 0.700923 \\ 8 & 2.581389 & -0.158237 & 1.220026 \\ 22 & 0.816229 & -0.128969 & 1.284754 \\ 8 & -0.291631 & -0.438779 & 2.757416 \\ 8 & 3.257293 & -2.031620 & -0.945130 \\ 8 & 0.813002 & 1.712145 & 0.850287 \\ 22 & -0.256413 & 2.967678 & -0.180670 \\ 8 & -2.014196 & 2.226495 & -0.539104 \\ 8 & 0.681880 & -1.929007 & 0.609034 \\ 8 & -0.309936 & 4.406461 & 0.544940 \\ 8 & 1.197882 & 2.811519 & -1.421271 \\ 8 & -2.519887 & -0.791166 & -1.415087 \\ 8 & -4.583062 & 0.741321 & -0.943020 \\ 8 & -5.202540 & -0.435404 & -1.142319 \\ 8 & -4.456519 & -1.486579 & -1.306467\end{array}$

Triplet bidentate (adsorption site \#3, isomer \#2)

$\begin{array}{rrrr}8 & 3.464070 & 1.965996 & -0.535548 \\ 22 & 4.081842 & 0.257167 & 0.070445 \\ 8 & 3.630324 & -1.147925 & -1.194610 \\ 22 & 1.897117 & -1.313476 & -1.289183 \\ 8 & 1.171924 & -1.918401 & 0.260353 \\ 22 & 0.823465 & -0.298465 & 1.232535 \\ 8 & 0.352099 & 1.527042 & 1.136245 \\ 22 & -0.946779 & 2.535234 & 0.134678 \\ 8 & 0.572267 & 3.075494 & -0.889909 \\ 22 & 1.721794 & 1.887489 & -0.273854 \\ 8 & 2.543381 & 0.155323 & 1.250527 \\ 8 & 1.152102 & 0.319447 & -1.100437 \\ 8 & 0.511359 & -2.291420 & -2.167999\end{array}$




$\begin{array}{rrrr}22 & -0.719329 & -2.205353 & -0.885493 \\ 8 & -2.365245 & -1.655569 & -1.498454 \\ 22 & -2.878637 & -0.376129 & -0.338006 \\ 8 & -4.641844 & 0.238134 & -0.896773 \\ 8 & -5.039856 & 1.547475 & -0.844342 \\ 8 & -4.125520 & 2.419859 & -0.644754 \\ 8 & -1.226324 & -3.160932 & 0.648848 \\ 22 & -1.548528 & -1.792803 & 1.738919 \\ 8 & -0.148116 & -1.079961 & 2.611834 \\ 8 & -0.972440 & -0.653774 & 0.260692 \\ 8 & -3.170440 & -1.077185 & 1.333454 \\ 8 & -2.329903 & 1.379786 & -0.565115 \\ 8 & 5.537566 & 0.205482 & 0.771640 \\ 8 & -1.600505 & 3.743552 & 0.991734\end{array}$

Triplet monodentate (adsorption site \#3, isomer \#1)

$\begin{array}{rrrr}8 & 3.378193 & 2.066547 & -0.715930 \\ 22 & 1.690074 & 1.842029 & -0.348416 \\ 8 & 1.163143 & 0.290028 & -1.144306 \\ 22 & 1.997542 & -1.329249 & -1.255249 \\ 8 & 0.656486 & -2.318639 & -2.106234 \\ 22 & -0.649970 & -2.170979 & -0.862525 \\ 8 & -1.141769 & -3.148389 & 0.653929 \\ 22 & -1.444656 & -1.783373 & 1.766984 \\ 8 & -0.822337 & -0.670520 & 0.332266 \\ 22 & -2.855085 & -0.304121 & -0.297104 \\ 8 & -3.030042 & -1.049714 & 1.421056 \\ 22 & 4.131324 & 0.407312 & -0.034947 \\ 8 & 5.590429 & 0.450122 & 0.658916 \\ 8 & 2.594867 & 0.360494 & 1.143858 \\ 22 & 0.904542 & -0.325706 & 1.269048 \\ 8 & -0.001709 & -1.070100 & 2.663891 \\ 8 & 3.731274 & -1.047902 & -1.233783 \\ 8 & 0.340559 & 1.530129 & 1.139617 \\ 22 & -0.956143 & 2.462969 & 0.213178 \\ 8 & -2.279530 & 1.424254 & -0.380500 \\ 8 & 1.371804 & -1.894601 & 0.343683 \\ 8 & -1.638019 & 3.842057 & 1.210842 \\ 8 & 0.365642 & 3.003757 & -0.894548 \\ 8 & -2.212021 & -1.579070 & -1.490398 \\ 8 & -4.557884 & -0.007876 & -0.827467 \\ 8 & -5.030181 & 1.516181 & -0.967195 \\ 8 & -6.227380 & 1.606318 & -1.047862\end{array}$

Triplet monodentate (adsorption site \#3, isomer \#2)

$\begin{array}{rrrr}8 & 3.848896 & 1.272561 & -0.919817 \\ 22 & 4.123982 & -0.297254 & -0.084152 \\ 8 & 3.520776 & -1.780745 & -0.977875 \\ 22 & 1.695675 & -1.788414 & -0.994586 \\ 8 & 1.029870 & -1.897182 & 0.650205 \\ 22 & 1.112872 & -0.111498 & 1.307972 \\ 8 & 1.112334 & 1.721590 & 0.815638 \\ 22 & -0.587176 & 2.324007 & -0.169448 \\ 8 & 0.837938 & 2.683909 & -1.506095 \\ 22 & 2.033325 & 1.665843 & -0.772368 \\ 8 & 2.936698 & -0.138722 & 1.292510 \\ 8 & 1.390221 & -0.021131 & -1.118893\end{array}$




$\begin{array}{rrrr}8 & 0.116317 & -2.413009 & -1.711402 \\ 22 & -1.064386 & -1.683823 & -0.509881 \\ 8 & -2.545774 & -1.078535 & -1.211943 \\ 22 & -3.164011 & 0.484739 & -0.363666 \\ 8 & -4.914703 & 0.698780 & -0.845882 \\ 8 & -5.643177 & -0.610781 & -0.345583 \\ 8 & -5.979313 & -1.328265 & -1.270786 \\ 8 & -1.673682 & -2.179250 & 1.184023 \\ 22 & -1.594576 & -0.587292 & 1.970679 \\ 8 & 0.040519 & -0.224825 & 2.761285 \\ 8 & -0.734747 & 0.013490 & 0.368161 \\ 8 & -3.038326 & 0.337538 & 1.509586 \\ 8 & -2.243276 & 1.810900 & -0.961204 \\ 8 & 5.869460 & -0.464585 & 0.469433 \\ 8 & -0.958221 & 3.580918 & 0.761129\end{array}$

Triplet bidentate (adsorption site \#4, isomer \#1)

$\begin{array}{rrrr}8 & 2.104714 & 2.862371 & -0.295889 \\ 22 & 0.530534 & 2.199426 & -0.730691 \\ 8 & 0.799117 & 0.406033 & -0.969835 \\ 22 & 1.787615 & -1.215218 & -0.800507 \\ 8 & 0.610995 & -2.202099 & -1.844333 \\ 22 & -0.832155 & -2.120282 & -0.770199 \\ 8 & -1.497805 & -3.017079 & 0.737928 \\ 22 & -2.126262 & -1.633575 & 1.671266 \\ 8 & -1.525107 & -0.542806 & 0.141062 \\ 22 & -3.239037 & -0.624152 & -0.679168 \\ 8 & -3.761209 & -1.213839 & 0.990364 \\ 22 & 3.043763 & 1.446708 & 0.570815 \\ 8 & 4.410841 & 1.854691 & 1.334771 \\ 8 & 1.668438 & 0.735807 & 1.719360 \\ 22 & 0.096366 & 0.176670 & 1.266598 \\ 8 & -0.996317 & -0.591411 & 2.583512 \\ 8 & 3.261726 & -0.016541 & -0.670682 \\ 8 & -0.647787 & 1.852343 & 0.716920 \\ 22 & -2.206025 & 2.424517 & -0.392041 \\ 8 & -3.390015 & 1.047492 & -1.055196 \\ 8 & 0.690815 & -1.564964 & 0.588334 \\ 8 & -2.979797 & 3.669555 & 0.279010 \\ 8 & -0.832783 & 2.937249 & -1.597616 \\ 8 & -2.385481 & -1.860842 & -1.723520 \\ 8 & 3.169416 & -2.582237 & -0.681802 \\ 8 & 4.499372 & -2.389903 & -0.365935 \\ 8 & 4.900163 & -1.182578 & -0.260654\end{array}$

Triplet bidentate (adsorption site \#4, isomer \#2)

$\begin{array}{rrrr}8 & 2.522059 & 1.808942 & -0.744413 \\ 22 & 3.041194 & 1.268586 & 0.888548 \\ 8 & 3.113367 & -0.507606 & 0.801309 \\ 22 & 1.911272 & -1.443180 & -0.223299 \\ 8 & 3.475188 & -2.857102 & -0.552675 \\ 8 & 3.912629 & -2.233746 & -1.629488 \\ 8 & 3.015956 & -1.251510 & -1.929905 \\ 22 & 0.908131 & 1.264420 & -1.445111 \\ 8 & -0.334168 & 1.919974 & -2.490244 \\ 22 & -1.799179 & 1.798872 & -1.211742 \\ 8 & -2.504647 & 3.177124 & -0.769366\end{array}$




$\begin{array}{rrrr}8 & 0.663760 & -0.522901 & -1.313350 \\ 22 & 0.055337 & 0.920300 & 1.592774 \\ 8 & 0.556607 & -0.720957 & 1.120118 \\ 22 & -0.779449 & -1.822416 & 0.063182 \\ 8 & -2.167174 & -2.136722 & -1.117799 \\ 22 & -3.276988 & -0.735938 & -0.823021 \\ 8 & -3.191035 & 0.618085 & -1.889794 \\ 8 & -0.145775 & 1.786842 & -0.014533 \\ 8 & 0.682020 & -2.838901 & -0.117328 \\ 8 & -1.730536 & -2.041665 & 1.671771 \\ 22 & -2.545197 & -0.490570 & 1.842728 \\ 8 & -1.463267 & 0.736764 & 2.639697 \\ 8 & -1.779016 & -0.016773 & 0.157339 \\ 8 & -4.079147 & -0.612292 & 0.849326 \\ 8 & 4.648259 & 2.016464 & 1.393541 \\ 8 & 1.638337 & 1.585779 & 2.054635\end{array}$

Triplet monodentate (adsorption site \#4, isomer \#1)

$\begin{array}{rrrr}8 & 1.483548 & 3.038549 & -0.582553 \\ 22 & -0.109826 & 2.138632 & -0.856900 \\ 8 & 0.358380 & 0.436565 & -1.059513 \\ 22 & 2.265054 & -0.998488 & -0.719617 \\ 8 & 1.126978 & -1.887689 & -1.901715 \\ 22 & -0.395260 & -1.650855 & -0.996090 \\ 8 & -0.860895 & -2.962395 & 0.327403 \\ 22 & -1.684908 & -1.994169 & 1.534704 \\ 8 & -1.579136 & -0.542880 & 0.257837 \\ 22 & -3.174148 & -1.139492 & -0.628237 \\ 8 & -3.411459 & -2.061484 & 0.965366 \\ 22 & 2.609972 & 1.973547 & 0.307634 \\ 8 & 4.036342 & 2.925019 & 0.978270 \\ 8 & 1.588448 & 1.175343 & 1.606764 \\ 22 & 0.090620 & 0.244539 & 1.286575 \\ 8 & -0.737169 & -0.787816 & 2.561786 \\ 8 & 3.141810 & 0.590288 & -0.720103 \\ 8 & -0.897877 & 1.826565 & 0.807006 \\ 22 & -2.722332 & 1.827298 & -0.040257 \\ 8 & -3.870449 & 0.425493 & -0.727974 \\ 8 & 0.991401 & -1.264043 & 0.574154 \\ 8 & -3.596188 & 2.958833 & 0.697701 \\ 8 & -1.702668 & 2.514045 & -1.504852 \\ 8 & -2.005443 & -1.732570 & -1.894945 \\ 8 & 3.626126 & -2.137107 & -0.374668 \\ 8 & 5.044784 & -1.417809 & -0.069063 \\ 8 & 5.845743 & -2.199692 & 0.367612\end{array}$

Triplet monodentate (adsorption site \#4, isomer \#2)

$\begin{array}{rrrr}8 & -2.460195 & -2.800853 & -0.389987 \\ 22 & -3.375945 & -1.314920 & 0.384449 \\ 8 & -3.410379 & 0.198041 & -0.830503 \\ 22 & -1.807710 & 0.862047 & -1.012785 \\ 8 & -2.820006 & 3.016926 & -0.620256 \\ 8 & -1.756079 & 3.893304 & -0.261207 \\ 8 & -1.911592 & 3.887940 & 0.987725 \\ 22 & -0.764463 & -2.329023 & -0.506609 \\ 8 & 0.526122 & -3.255245 & -1.270373 \\ 22 & 1.967817 & -2.669057 & -0.159488\end{array}$




$\begin{array}{rrrr}8 & 2.694468 & -3.871223 & 0.632629 \\ 8 & -0.689671 & -0.586163 & -1.117607 \\ 22 & -0.203736 & -0.178088 & 1.262200 \\ 8 & -0.780520 & 1.447443 & 0.394640 \\ 22 & 0.738933 & 1.869193 & -0.974789 \\ 8 & 2.319078 & 1.451935 & -1.852490 \\ 22 & 3.120158 & 0.337981 & -0.660585 \\ 8 & 3.207590 & -1.370862 & -0.874905 \\ 8 & 0.487574 & -1.914534 & 0.907042 \\ 8 & -0.668665 & 1.991598 & -2.066491 \\ 8 & 1.395869 & 2.924452 & 0.441486 \\ 22 & 1.993337 & 1.647107 & 1.535900 \\ 8 & 0.811058 & 0.730666 & 2.545631 \\ 8 & 1.414717 & 0.395407 & 0.156161 \\ 8 & 3.632007 & 1.139005 & 0.936800 \\ 8 & -4.730466 & -1.638236 & 1.207229 \\ 8 & -1.848983 & -0.759016 & 1.436671\end{array}$

Triplet bidentate (adsorption site \#5, isomer \#1)

$\begin{array}{rrrr}8 & -3.114615 & 1.265232 & -0.632121 \\ 22 & -1.509364 & 1.553975 & 0.198854 \\ 8 & -0.886153 & -0.074772 & 0.808268 \\ 22 & -1.071649 & -1.872464 & 0.900202 \\ 8 & 0.336428 & -2.350223 & 2.108473 \\ 22 & 1.712967 & -1.776430 & 1.136776 \\ 8 & 2.795409 & -2.469995 & -0.226540 \\ 22 & 2.838201 & -1.072758 & -1.340701 \\ 8 & 1.656990 & -0.203036 & -0.027669 \\ 22 & 3.106304 & 0.669992 & 0.822022 \\ 8 & 4.021494 & 0.152243 & -0.689319 \\ 22 & -3.229454 & -0.736283 & -0.887325 \\ 8 & -4.444364 & -1.178237 & -1.841083 \\ 8 & -1.581114 & -0.634206 & -1.899475 \\ 22 & 0.034110 & -0.390643 & -1.351538 \\ 8 & 1.497302 & -0.762850 & -2.472618 \\ 8 & -2.714906 & -2.124468 & 0.387674 \\ 8 & -0.091360 & 1.507251 & -1.079395 \\ 22 & 0.902727 & 2.896349 & -0.098603 \\ 8 & 2.426133 & 2.254210 & 0.894321 \\ 8 & 0.115666 & -2.098299 & -0.432276 \\ 8 & 1.215438 & 4.180679 & -1.021075 \\ 8 & -0.750730 & 2.996789 & 0.857517 \\ 8 & 2.858546 & -0.670799 & 2.050387 \\ 8 & -4.400702 & 0.382301 & 0.805741 \\ 8 & -3.805209 & 0.517969 & 2.022296 \\ 8 & -2.789820 & 1.312931 & 2.092753\end{array}$

Triplet bidentate (adsorption site \#5, isomer \#2)

$\begin{array}{rrrr}8 & -2.849193 & 1.689223 & 0.231857 \\ 22 & -3.513510 & -0.002642 & -0.542263 \\ 8 & -3.357766 & -1.591803 & 0.551258 \\ 22 & -1.652810 & -1.909651 & 0.827982 \\ 8 & -0.656043 & -2.198548 & -0.624032 \\ 22 & -0.473459 & -0.438445 & -1.419987 \\ 8 & -0.219738 & 1.464325 & -1.012727 \\ 22 & 1.134890 & 2.778581 & -0.555257 \\ 8 & -0.139265 & 3.293155 & 0.823038\end{array}$




$\begin{array}{rrrr}22 & -1.186489 & 1.627962 & 0.607053 \\ 8 & -0.825513 & 1.428172 & 2.860232 \\ 8 & 0.426721 & 1.103758 & 2.876339 \\ 8 & 1.045939 & 1.417281 & 1.682000 \\ 8 & -1.034237 & -0.197862 & 0.812012 \\ 8 & -0.293301 & -2.542150 & 1.990615 \\ 22 & 1.111914 & -1.985826 & 1.022834 \\ 8 & 2.430947 & -1.051329 & 1.813397 \\ 22 & 2.764143 & 0.260036 & 0.476684 \\ 8 & 2.755261 & 1.931074 & -0.068599 \\ 8 & 1.954709 & -2.919642 & -0.352425 \\ 22 & 2.171873 & -1.596899 & -1.536330 \\ 8 & 0.851386 & -1.026789 & -2.621778 \\ 8 & 1.220789 & -0.511440 & -0.249910 \\ 8 & 3.576946 & -0.628869 & -0.907194 \\ 8 & -4.975222 & 0.151439 & -1.198292 \\ 8 & -2.140483 & -0.277054 & -1.858668 \\ 8 & 1.247544 & 3.950985 & -1.669089\end{array}$

\section{Triplet monodentate (adsorption site \#5, isomer \#1)}

$\begin{array}{rrrr}8 & 3.472589 & -0.376008 & -0.977920 \\ 22 & 2.248025 & 0.789167 & -0.570371 \\ 8 & 0.690121 & -0.468479 & -1.058776 \\ 22 & 0.414388 & -2.228357 & -0.964718 \\ 8 & -1.321461 & -2.628336 & -1.680590 \\ 22 & -2.315368 & -1.728209 & -0.533144 \\ 8 & -3.140762 & -1.775222 & 1.171704 \\ 22 & -2.634599 & -0.188619 & 1.794713 \\ 8 & -1.645867 & -0.033759 & 0.106700 \\ 22 & -2.971105 & 1.012220 & -0.715854 \\ 8 & -3.592703 & 1.179508 & 0.995619 \\ 22 & 3.111210 & -1.825139 & 0.221197 \\ 8 & 4.363033 & -2.473743 & 1.009465 \\ 8 & 1.925534 & -0.613002 & 1.225152 \\ 22 & 0.173895 & -0.273228 & 1.107203 \\ 8 & -1.039016 & -0.098495 & 2.552867 \\ 8 & 1.941636 & -3.036365 & -0.696928 \\ 8 & 0.374029 & 1.513568 & 0.594675 \\ 22 & -0.057940 & 2.418463 & -0.928650 \\ 8 & -1.843584 & 2.130381 & -1.466300 \\ 8 & -0.411216 & -2.056515 & 0.651481 \\ 8 & 0.072865 & 4.210134 & -0.650708 \\ 8 & 1.402955 & 1.979507 & -1.799234 \\ 8 & -3.426582 & -0.580276 & -1.458545 \\ 8 & 3.200695 & 2.004025 & 0.426390 \\ 8 & 2.417933 & 3.293932 & 0.744326 \\ 8 & 2.146413 & 3.394325 & 1.932091\end{array}$

Triplet monodentate (adsorption site \#5, isomer \#2)

$\begin{array}{rrrr}8 & 3.151341 & -0.058146 & -1.366841 \\ 22 & 3.069934 & -1.430097 & -0.041952 \\ 8 & 2.064158 & -2.843866 & -0.838822 \\ 22 & 0.471041 & -2.137877 & -1.091067 \\ 8 & -0.304294 & -2.033030 & 0.540245 \\ 22 & 0.184116 & -0.245006 & 1.082274 \\ 8 & 0.331191 & 1.543654 & 0.561225 \\ 22 & -0.518648 & 2.770470 & -0.679420\end{array}$




$\begin{array}{rrrr}8 & 1.074853 & 2.437074 & -1.693291 \\ 22 & 1.847182 & 1.259298 & -0.667509 \\ 8 & 3.240046 & 2.111965 & 0.165911 \\ 8 & 4.201125 & 1.145190 & 0.969708 \\ 8 & 4.142325 & 1.333165 & 2.164661 \\ 8 & 0.755555 & -0.372464 & -1.160443 \\ 8 & 1.941647 & -0.477623 & 1.188817 \\ 8 & -1.251364 & -2.560368 & -1.791144 \\ 22 & -2.273952 & -1.757105 & -0.581239 \\ 8 & -3.503329 & -0.672365 & -1.383998 \\ 22 & -3.183181 & 0.915435 & -0.519459 \\ 8 & -2.303178 & 2.224922 & -1.204766 \\ 8 & -2.999339 & -1.990950 & 1.145166 \\ 22 & -2.581638 & -0.417972 & 1.867345 \\ 8 & -0.958175 & -0.212907 & 2.567539 \\ 8 & -1.689765 & -0.054321 & 0.141695 \\ 8 & -3.696657 & 0.891174 & 1.249073 \\ 8 & 4.556528 & -1.795424 & 0.527934 \\ 8 & -0.543517 & 4.252169 & -0.047342\end{array}$

Triplet bidentate (adsorption site \#6, isomer \#1)

$\begin{array}{rrrr}8 & 3.126179 & 1.652788 & -0.940850 \\ 22 & 1.281520 & 1.594614 & -0.654084 \\ 8 & 0.581656 & 0.238366 & -1.677596 \\ 22 & 1.413667 & -1.348375 & -1.344236 \\ 8 & 0.131274 & -2.226816 & -2.342147 \\ 22 & -1.246859 & -1.612272 & -1.311909 \\ 8 & -2.278056 & -2.619988 & -0.148718 \\ 22 & -2.546195 & -1.426181 & 1.162007 \\ 8 & -1.518993 & -0.194250 & 0.066373 \\ 22 & -3.073237 & 0.497422 & -0.767480 \\ 8 & -3.925083 & -0.352258 & 0.634139 \\ 22 & 3.653983 & 0.053191 & -0.345755 \\ 8 & 5.228075 & -0.073515 & 0.615131 \\ 8 & 2.058388 & -0.196744 & 0.498009 \\ 22 & 0.202097 & -0.387825 & 1.330905 \\ 8 & -1.237323 & -1.121470 & 2.321809 \\ 8 & 3.230487 & -1.218155 & -1.581713 \\ 8 & 0.145648 & 1.448158 & 0.903916 \\ 22 & -1.084072 & 2.811982 & 0.188967 \\ 8 & -2.688434 & 2.182201 & -0.641097 \\ 8 & 0.260610 & -1.810422 & 0.058727 \\ 8 & -1.289460 & 4.054337 & 1.196887 \\ 8 & 0.358220 & 3.021143 & -1.048740 \\ 8 & -2.561907 & -0.590574 & -2.129955 \\ 8 & 1.098339 & 0.190283 & 3.015635 \\ 8 & 1.689109 & -0.976027 & 3.434262 \\ 8 & 1.438784 & -1.909089 & 2.555290\end{array}$

\section{Triplet monodentate (adsorption site \#6, isomer \#1)}

$\begin{array}{rrrr}8 & 3.125351 & 1.106323 & -1.224026 \\ 22 & 1.277462 & 1.193140 & -1.042068 \\ 8 & 0.442413 & -0.327482 & -1.671789 \\ 22 & 1.219378 & -1.781044 & -0.891809 \\ 8 & -0.165627 & -2.826662 & -1.595016 \\ 22 & -1.460252 & -1.871296 & -0.765467 \\ 8 & -2.541984 & -2.455408 & 0.641354\end{array}$




$\begin{array}{rrrr}22 & -2.651016 & -0.959436 & 1.599465 \\ 8 & -1.608440 & -0.114395 & 0.196043 \\ 22 & -3.154770 & 0.418833 & -0.756820 \\ 8 & -3.988449 & 0.028198 & 0.851381 \\ 22 & 3.583356 & -0.365252 & -0.306179 \\ 8 & 5.288578 & -0.567174 & 0.384226 \\ 8 & 1.888079 & -0.415239 & 0.456216 \\ 22 & 0.151474 & -0.116829 & 1.419990 \\ 8 & -1.247990 & -0.490101 & 2.614580 \\ 8 & 3.045060 & -1.808651 & -1.250509 \\ 8 & 0.237113 & 1.547173 & 0.534572 \\ 22 & -0.975951 & 2.733562 & -0.490908 \\ 8 & -2.653104 & 2.045472 & -1.094529 \\ 8 & 0.033573 & -1.845999 & 0.605894 \\ 8 & -1.061477 & 4.206352 & 0.159574 \\ 8 & 0.424501 & 2.524424 & -1.777562 \\ 8 & -2.755187 & -1.008962 & -1.795477 \\ 8 & 1.289665 & 0.377428 & 2.837248 \\ 8 & 2.356499 & 1.315052 & 2.438742 \\ 8 & 3.419801 & 0.767538 & 2.082015\end{array}$

\section{Optimized structures of the transition states}

Triplet monodentate (adsorption site \#1, isomer \#2)

$\begin{array}{rrrr}8 & 4.029501 & -0.234969 & -0.731812 \\ 22 & 2.579429 & 0.912802 & -0.593867 \\ 8 & 1.183780 & -0.153062 & -1.113686 \\ 22 & 0.677486 & -0.002176 & 1.304178 \\ 8 & -0.905099 & 0.620767 & 0.322460 \\ 22 & -2.150813 & 1.643024 & -0.605582 \\ 8 & -1.012899 & 2.784722 & -1.282127 \\ 22 & 0.737061 & 2.976988 & -0.541245 \\ 8 & 0.958276 & 4.457679 & 0.052181 \\ 22 & 3.514486 & -1.681132 & 0.210342 \\ 8 & 4.877780 & -2.580267 & 1.068002 \\ 8 & 2.371426 & -2.728031 & -0.710233 \\ 22 & 0.735919 & -1.879990 & -0.873978 \\ 22 & -2.056984 & -1.330702 & -0.493183 \\ 8 & -2.784217 & 0.122731 & -1.358389 \\ 8 & 2.386590 & -0.719809 & 1.294166 \\ 8 & -0.065703 & -1.687260 & 0.714024 \\ 8 & -0.837895 & -2.055106 & -1.703771 \\ 8 & 1.451988 & 1.663014 & 0.762001 \\ 22 & -2.006840 & 0.521070 & 1.905105 \\ 8 & -2.878316 & 1.901872 & 1.063622 \\ 8 & -0.349867 & 0.305894 & 2.741258 \\ 8 & 2.296386 & 2.340099 & -1.534824 \\ 8 & -3.263973 & -2.629555 & -0.726245 \\ 8 & -4.804379 & -1.952537 & -0.098626 \\ 8 & -5.557116 & -1.694408 & -0.978147 \\ 8 & -2.678061 & -0.951454 & 1.362775\end{array}$

Triplet monodentate (adsorption site \#3, isomer \#2)

$\begin{array}{rrrr}8 & 3.855908 & 1.229232 & -0.923844 \\ 22 & 2.043954 & 1.641557 & -0.782897 \\ 8 & 1.379774 & -0.039289 & -1.117361\end{array}$




$\begin{array}{rrrr}22 & 1.096788 & -0.103774 & 1.310674 \\ 22 & -1.615780 & -0.552613 & 1.966546 \\ 8 & -3.047198 & 0.383151 & 1.507349 \\ 22 & -3.160121 & 0.535507 & -0.375455 \\ 8 & -2.226251 & 1.859611 & -0.968653 \\ 22 & -0.564544 & 2.353735 & -0.185121 \\ 8 & -0.907516 & 3.632649 & 0.726809 \\ 22 & 4.113272 & -0.334559 & -0.071161 \\ 8 & 5.855571 & -0.514138 & 0.491047 \\ 8 & 3.497423 & -1.818594 & -0.955248 \\ 22 & 1.671880 & -1.808090 & -0.974879 \\ 22 & -1.086958 & -1.658252 & -0.509736 \\ 8 & -0.745399 & 0.037204 & 0.366648 \\ 8 & 2.922320 & -0.151656 & 1.297690 \\ 8 & 0.998287 & -1.894141 & 0.667960 \\ 8 & 0.091400 & -2.421179 & -1.696152 \\ 8 & 1.122323 & 1.725736 & 0.803887 \\ 8 & 0.866154 & 2.671739 & -1.527126 \\ 8 & -2.556619 & -1.042411 & -1.223848 \\ 8 & -1.713535 & -2.145722 & 1.178692 \\ 8 & -4.886858 & 0.759021 & -0.866978 \\ 8 & -5.543586 & -0.788822 & -0.323129 \\ 8 & -5.855258 & -1.485787 & -1.239174 \\ 8 & 0.022205 & -0.198758 & 2.762008\end{array}$

Triplet monodentate (adsorption site \#4, isomer \#1)

$\begin{array}{rrrr}8 & 1.782519 & 2.846478 & -0.618576 \\ 22 & 2.797760 & 1.704892 & 0.308600 \\ 8 & 3.204477 & 0.238721 & -0.651244 \\ 22 & 2.177389 & -1.269944 & -0.631340 \\ 8 & 3.387217 & -2.550088 & -0.285462 \\ 8 & 4.892987 & -1.784088 & 0.401629 \\ 8 & 5.643333 & -1.394058 & -0.426426 \\ 22 & 0.106323 & 2.101275 & -0.896314 \\ 8 & -1.455870 & 2.558794 & -1.565610 \\ 22 & -2.527105 & 1.900554 & -0.117273 \\ 8 & -3.341592 & 3.043176 & 0.669302 \\ 8 & 0.426402 & 0.356293 & -1.026769 \\ 22 & 0.160833 & 0.274152 & 1.316040 \\ 8 & -0.696361 & 1.894586 & 0.766920 \\ 22 & -0.544090 & -1.635124 & -0.922192 \\ 8 & -2.115411 & -1.677034 & -1.873281 \\ 22 & -3.301041 & -0.943128 & -0.689985 \\ 8 & -3.823677 & 0.670793 & -0.909426 \\ 8 & 0.959389 & -2.061347 & -1.794363 \\ 8 & 0.872136 & -1.343657 & 0.663945 \\ 8 & -1.157517 & -2.824637 & 0.455764 \\ 22 & -1.930583 & -1.725202 & 1.577516 \\ 8 & -0.863432 & -0.594832 & 2.570133 \\ 8 & -1.699491 & -0.373722 & 0.220872 \\ 8 & -3.649478 & -1.710948 & 0.965512 \\ 8 & 4.324801 & 2.526484 & 0.927525 \\ 8 & 1.725986 & 1.058533 & 1.660664\end{array}$

Triplet monodentate (adsorption site \#5, isomer \#1)

$\begin{array}{rrrr}8 & 3.500123 & -0.391426 & -0.944174 \\ 22 & 3.105853 & -1.838281 & 0.232767\end{array}$




$\begin{array}{rrrr}8 & 1.928092 & -3.007822 & -0.726923 \\ 22 & 0.413337 & -2.176339 & -0.988564 \\ 8 & -0.402721 & -2.032636 & 0.642076 \\ 22 & 0.186286 & -0.253917 & 1.107722 \\ 8 & 0.412119 & 1.529944 & 0.605620 \\ 22 & -0.075932 & 2.245995 & -1.006342 \\ 8 & 1.464809 & 1.959555 & -1.799290 \\ 22 & 2.323101 & 0.821861 & -0.511149 \\ 8 & 3.330147 & 1.967205 & 0.443352 \\ 8 & 2.326257 & 3.480645 & 0.764708 \\ 8 & 2.004928 & 3.577636 & 1.900600 \\ 8 & 0.677136 & -0.412702 & -1.062309 \\ 8 & 1.933801 & -0.611308 & 1.233866 \\ 8 & -1.324492 & -2.600628 & -1.672766 \\ 22 & -2.307671 & -1.733446 & -0.487515 \\ 8 & -3.465252 & -0.628769 & -1.406228 \\ 22 & -3.016008 & 0.987199 & -0.721278 \\ 8 & -1.889580 & 2.037622 & -1.551327 \\ 8 & -3.116097 & -1.751661 & 1.231113 \\ 22 & -2.618723 & -0.149988 & 1.812963 \\ 8 & -1.014106 & -0.057934 & 2.557040 \\ 8 & -1.650788 & -0.013693 & 0.110155 \\ 8 & -3.596049 & 1.198049 & 1.006592 \\ 8 & 4.341725 & -2.517683 & 1.023173 \\ 8 & 0.011775 & 4.042127 & -0.811443\end{array}$

